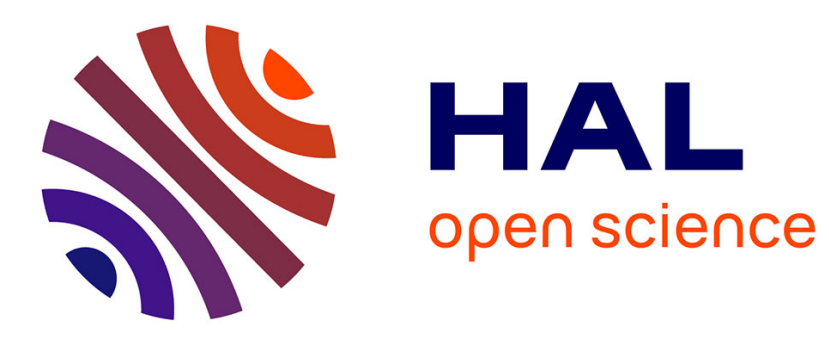

\title{
Computational Homogenization of Architectured Materials
}

\author{
Justin Dirrenberger, Samuel Forest, Dominique Jeulin
}

\section{To cite this version:}

Justin Dirrenberger, Samuel Forest, Dominique Jeulin. Computational Homogenization of Architectured Materials. Architectured Materials in Nature and Engineering, Springer, pp.89-139, 2019, 9781493991587. 10.1007/978-3-030-11942-3_4. hal-02129186

\section{HAL Id: hal-02129186 \\ https://hal.science/hal-02129186}

Submitted on 14 May 2019

HAL is a multi-disciplinary open access archive for the deposit and dissemination of scientific research documents, whether they are published or not. The documents may come from teaching and research institutions in France or abroad, or from public or private research centers.
L'archive ouverte pluridisciplinaire HAL, est destinée au dépôt et à la diffusion de documents scientifiques de niveau recherche, publiés ou non, émanant des établissements d'enseignement et de recherche français ou étrangers, des laboratoires publics ou privés. 


\title{
Computational Homogenization of Architectured Materials
}

\author{
Justin Dirrenberger, Samuel Forest and Dominique Jeulin
}

\begin{abstract}
Architectured materials involve geometrically engineered distributions of microstructural phases at a scale comparable to the scale of the component, thus calling for new models in order to determine the effective properties of materials. The present chapter aims at providing such models, in the case of mechanical properties. As a matter of fact, one engineering challenge is to predict the effective properties of such materials; computational homogenization using finite element analysis is a powerful tool to do so. Homogenized behavior of architectured materials can thus be used in large structural computations, hence enabling the dissemination of architectured materials in the industry. Furthermore, computational homogenization is the basis for computational topology optimization which will give rise to the next generation of architectured materials. This chapter covers the computational homogenization of periodic architectured materials in elasticity and plasticity, as well as the homogenization and representativity of random architectured materials.
\end{abstract}

\subsection{Introduction}

Architectured materials are a rising class of materials that bring new possibilities in terms of functional properties, filling the gaps and pushing the limits of Ashby's materials performance maps [11]. The term architectured materials encompasses

\author{
J. Dirrenberger $(\bowtie)$ \\ Laboratoire PIMM, Arts et Métiers-ParisTech, CNRS, Cnam, \\ 151 boulevard de l'Hôpital, 75013 Paris, France \\ e-mail: justin.dirrenberger@ensam.eu \\ S. Forest \\ Centre des Matériaux, MINES-ParisTech, CNRS UMR 7633, BP 87, \\ 91003 Evry cedex, France \\ e-mail: samuel.forest@mines-paristech.fr \\ D. Jeulin \\ Centre de Morphologie Mathématique, MINES-ParisTech, 35 rue St-Honoré, \\ 77300 Fontainebleau, France \\ e-mail: dominique.jeulin@mines-paristech.fr
}


any microstructure designed in a thoughtful fashion, such that some of its materials properties, e.g. yield strength/density, have been improved in comparison to those of its constituents, due to both structure and composite effects, which depend on the multiphase morphology, i.e. the relative topological arrangement between each phase $[11,33,35]$.

There are many examples: particulate and fibrous composites, foams, sandwich structures, woven materials, lattice structures, etc. with different objectives. For instance, developing architectured porous materials for structural, acoustic and insulation properties [40, 70], entangled monofilament of pearlitic steel [47, 173], sandwich composite structures [118, 163-165], segmented interlocking structures [59, $60,62,65,66,72,117,123,136,145-147,189]$, asymmetric frictional materials $[18,19]$, woven and non-woven textile composites [57, 130, 141], porous metallic glasses [187], hierarchical composites [92], crumpled metallic foils [34]. Much more examples can be found in the present book.

One can play on many parameters in order to obtain architectured materials, but all of them are related either to the microstructure or the geometry. Parameters related to the microstructure can be optimised for specific needs using a materials-by-design approach, which has been thoroughly developed by chemists, materials scientists and metallurgists. Properties improvements related to microstructural design are intrinsically linked to the synthesis and processing of materials and are therefore due to micro and nanoscale phenomena, taking place at a scale ranging from $1 \mathrm{~nm}$ to $10 \mu \mathrm{m}$. This scale is below the scope of the present chapter, but has been extensively studied in the literature [63, 78, 148].

From a macroscopic viewpoint, parameters related to the geometry have mainly been the responsibility of structural and civil engineers for centuries: to efficiently distribute materials within structures. An obvious example would be the many different strategies available for building bridges. At the millimetre scale, materials can be considered as structures, i.e. one can enhance the bending stiffness of a component by modifying its geometry while keeping the lineic mass (for beams) or surfacic mass (for plates) unchanged [209]. On the other hand, one might need a lower flexural strength for specific applications, with the same lineic and/or surfacic masses. This can be achieved with strand structures, i.e. by creating topological interfaces in the material. Processing remains the key technological issue for further development of architectured materials as the microstructure, the shape, and the scale of the material depend on it. Progress is made every day in terms of material processing at the lab scale, as it was done in [191] by using a bottom-up approach of sequential processing techniques in order to fabricate ultralight metallic microlattice materials [190]. There is still a long way to go for the industry to actually apply architectured materials in product manufacturing.

Architectured materials lie between the microscale and the macroscale. This class of materials involves geometrically engineered distributions of microstructural phases at a scale comparable to the scale of the component $[11,33,35]$, thus calling for enriched models of continuum mechanics in order to determine the 
effective properties of materials [81, 140], e.g. generalised continua theories, in order to describe the behaviour of architectured materials, such as strain-gradient elasticity [13], and strain-gradient plasticity. This topic has been especially fruitful these last few years in the mechanics of materials community $[9,13,45,51,129,166,167$, $174,203]$; this results in the availability of versatile models able to describe the various situations encountered with architectured materials. Given mature processing techniques, architectured materials are promised to a bright future in industrial applications due to their enticing customisable specific properties and the opportunity for multifunctionality.

When considering actual applications, one engineering challenge is to predict the effective properties of such materials; computational homogenisation using finite element analysis is a powerful tool to do so. Homogenised behaviour of architectured materials can thus be used in large structural computations, hence enabling the dissemination of architectured materials in the industry. Furthermore, computational homogenisation is the basis for computational topology optimisation [3, 10, 24, 41, $86,116,184,205,208,210,211]$ which will give rise to the next generation of architectured materials as it can already be seen in the works of $[8,48,71,83,96$, 119, 120, 128, 132, 149, 171, 204, 207].

Therefore, the development of architectured materials is related to the availability of appropriate computational tools for both design and modelling, but also for computerised manufacturing as for the various additive manufacturing techniques considered for producing architectured materials. In order to foster the development of architectured materials within an industrial framework, the availability of modelling bricks describing the underlying behaviour of such materials, is a necessity in order for these to be used in structural simulation codes. We are aiming at developing product design methodologies taking into account the specificities of architectured materials. To do so, we focused on computational approaches to modelling and optimising architectured materials, as well as novel processing techniques.

Materials science comes from the following fact: microstructural heterogeneities play a critical role in the macroscopic behaviour of a material $[29,32,77,112$, $151,202]$. Constitutive modelling, thanks to an interaction between experiments and simulation, is usually able to describe the response of most materials in use. Such phenomenological models, including little to no information about the microstructure, cannot necessarily account for local fluctuation of properties. In this case, the material is considered as a homogeneous medium. Studying the behaviour of heterogeneous materials involves developing enriched models including morphological information about the microstructure $[22$, 49, 64, 105, 108, 115, 159, 195, 197, 201, 213]. These models should be robust enough to predict effective properties depending on statistical data (volume fraction, $n$-point correlation function, etc.) and the physical nature of each phase or constituent. As a matter of fact, advanced models are often restricted to a limited variety of materials. Although isotropic and anisotropic polycrystalline metals, for instance, have been extensively studied by the means of both analytical and computational tools [25, 27, 38, 79, 97, 114, 121, 133, 157], some 
material configurations (architectured materials, materials with infinite contrast of properties, nanocomposites, materials exhibiting nonlinear behaviour, etc.) call for further development of models and tools for describing their effective behaviour. The purpose of this chapter is to present existing homogenization methods applicable to architectured materials.

The chapter was conceived as a basis for the enquiring reader to find out the main concepts related to computational homogenization of architectured materials, as well as the bibliographical references necessary to further his/her understanding of the present topic. It is based on the Ph.D. dissertation of the first author [54]. The chapter is organized as follows: firstly, classical homogenization for linear elasticity is presented in Sect. 4.2 and applied to the case of periodic architectured materials with negative Poisson's ratio, or auxetics. For a similar case of application, computational homogenization for elastoplasticity is introduced in Sect. 4.3. Since architectured materials are not always periodic, considerations regarding the of random media based on computational homogenization are presented in Sect. 4.4. Conclusions and perspectives are postponed to Sect. 4.5.

In this chapter, zeroth, first, and second order tensors are denoted by $a, \underline{\boldsymbol{a}}, \boldsymbol{a}$ respectively. The simple and double contractions are written . and : respectively. In index form with respect to an orthonormal Cartesian basis, these notations correspond to

$$
\underline{\boldsymbol{a}} \cdot \underline{\boldsymbol{b}}=a_{i} b_{j}, \quad \underset{\sim}{\boldsymbol{a}}: \underset{\sim}{\boldsymbol{b}}=a_{i j} b_{i j}
$$

where repeated indices are summed up. The tensor product is denoted by $\otimes$. For example, the component $(\underset{\sim}{\boldsymbol{a}} \otimes \underset{\sim}{\boldsymbol{b}})_{i j k l}$ is $a_{i j} b_{k l}$. The small strain assumption is made in this chapter. The nabla operator is denoted by $\nabla_{X}\left(\right.$ resp. $\left.\nabla_{x}\right)$ when partial derivation is computed with respect to macroscopic (resp. microscopic) coordinates. For example $\underset{\sim}{\sigma} . \boldsymbol{\nabla}$ is the divergence of the second order tensor $\underset{\sim}{\boldsymbol{\sigma}}$. The index form of $\underset{\sim}{\boldsymbol{\sigma}} . \boldsymbol{\nabla}$ is $\sigma_{i j, j}$. Similarly, $\underline{\boldsymbol{u}} \otimes \nabla$ means $u_{i, j}$. The sign $:=$ defines the quantity on the left-hand side.

\subsection{Computational Homogenization for Linear Elasticity}

\subsubsection{Constitutive Equations}

When heterogeneous materials are assumed to respond linearly to mechanical loading, constitutive relations are expressed locally for each phase in a linear elasticity framework using the generalized Hooke law:

$$
\underset{\sim}{\sigma}=\underset{\sim}{\boldsymbol{c}}: \underset{\sim}{\varepsilon}
$$

with $\underset{\sim}{\sigma}$ second-order symmetric Cauchy stress tensor, $\underset{\sim}{\boldsymbol{\varepsilon}}$ second-order symmetric engineering strain tensor and $\boldsymbol{c}$, fourth-order positive definite tensor of elastic moduli, 
also known as the elastic stiffness tensor. It is possible to express strain as a function of stress using the Compliance tensor $\underset{\sim}{s}$, which is defined as the inverse of tensor $\underset{\sim}{c}$ :

$$
\underset{\sim}{\varepsilon}=\underset{\approx}{\boldsymbol{s}}: \underset{\sim}{\boldsymbol{\sigma}} \text { with, } \underset{\approx}{\boldsymbol{s}}:=\boldsymbol{c}^{-1}
$$

such that,

$$
\underset{\approx}{\boldsymbol{s}} \cdot \underset{\approx}{\boldsymbol{c}}=\underset{\approx}{\boldsymbol{I}}
$$

with $\underset{\approx}{\boldsymbol{I}}$, fourth-order identity tensor operating on symmetric second-order tensors such that:

$$
\underset{\approx}{\boldsymbol{I}}=\frac{1}{2}\left(\delta_{i k} \delta_{j l}+\delta_{i l} \delta_{j k}\right) \underline{\boldsymbol{e}}_{i} \otimes \underline{\boldsymbol{e}}_{j} \otimes \underline{\boldsymbol{e}}_{k} \otimes \underline{\boldsymbol{e}}_{l}
$$

The 81 components of $c_{i j k l}$ can be thinned-down to 21 for the most anisotropic case (triclinic elasticity) due to symmetries of $\underset{\sim}{\boldsymbol{\sigma}}$ and $\underset{\sim}{\boldsymbol{\varepsilon}}$. By isomorphism, these 21 components can be written as a symmetric second-order tensor (matrix) $c_{I J}$ with 21 independent components using Voigt's notation:

$$
\left[\begin{array}{l}
\sigma_{11} \\
\sigma_{22} \\
\sigma_{33} \\
\sigma_{23} \\
\sigma_{31} \\
\sigma_{12}
\end{array}\right]=\left[\begin{array}{cccccc}
c_{11} & c_{12} & c_{13} & c_{14} & c_{15} & c_{16} \\
\bullet & c_{22} & c_{23} & c_{24} & c_{25} & c_{26} \\
\bullet & \bullet & c_{33} & c_{34} & c_{35} & c_{36} \\
\bullet & \bullet & \bullet & c_{44} & c_{45} & c_{46} \\
\bullet & \bullet & \bullet & \bullet & c_{55} & c_{56} \\
\bullet & \bullet & \bullet & \bullet & \bullet & c_{66}
\end{array}\right]\left[\begin{array}{c}
\varepsilon_{11} \\
\varepsilon_{22} \\
\varepsilon_{33} \\
\gamma_{23} \\
\gamma_{31} \\
\gamma_{12}
\end{array}\right]
$$

Engineering shear strain is used in the strain column-vector:

$$
\begin{aligned}
\gamma_{23} & =2 \varepsilon_{23} \\
\gamma_{31} & =2 \varepsilon_{31} \\
\gamma_{12} & =2 \varepsilon_{12}
\end{aligned}
$$

The matrix form of the compliance tensor is obtained by inverting (4.6):

$$
\left[\begin{array}{c}
\varepsilon_{11} \\
\varepsilon_{22} \\
\varepsilon_{33} \\
\gamma_{23} \\
\gamma_{31} \\
\gamma_{12}
\end{array}\right]=\left[\begin{array}{cccccc}
s_{11} & s_{12} & s_{13} & s_{14} & s_{15} & s_{16} \\
\bullet & s_{22} & s_{23} & s_{24} & s_{25} & s_{26} \\
\bullet & \bullet & s_{33} & s_{34} & s_{35} & s_{36} \\
\bullet & \bullet & \bullet & s_{44} & s_{45} & s_{46} \\
\bullet & \bullet & \bullet & \bullet & s_{55} & s_{56} \\
\bullet & \bullet & \bullet & \bullet & \bullet & s_{66}
\end{array}\right]\left[\begin{array}{l}
\sigma_{11} \\
\sigma_{22} \\
\sigma_{33} \\
\sigma_{23} \\
\sigma_{31} \\
\sigma_{12}
\end{array}\right]
$$


The finite element code used in the applications is actually making use of the Mandel notation presented in (4.8) and (4.9):

$$
\left[\begin{array}{c}
\sigma_{11} \\
\sigma_{22} \\
\sigma_{33} \\
\sqrt{2} \sigma_{23} \\
\sqrt{2} \sigma_{31} \\
\sqrt{2} \sigma_{12}
\end{array}\right]=\left[\begin{array}{cccccc}
c_{11} & c_{12} & c_{13} & \sqrt{2} c_{14} & \sqrt{2} c_{15} & \sqrt{2} c_{16} \\
\bullet & c_{22} & c_{23} & \sqrt{2} c_{24} & \sqrt{2} c_{25} & \sqrt{2} c_{26} \\
\bullet & \bullet & c_{33} & \sqrt{2} c_{34} & \sqrt{2} c_{35} & \sqrt{2} c_{36} \\
\bullet & \bullet & \bullet & 2 c_{44} & 2 c_{45} & 2 c_{46} \\
\bullet & \bullet & \bullet & \bullet & 2 c_{55} & 2 c_{56} \\
\bullet & \bullet & \bullet & \bullet & \bullet & 2 c_{66}
\end{array}\right]\left[\begin{array}{c}
\varepsilon_{11} \\
\varepsilon_{22} \\
\varepsilon_{33} \\
\sqrt{2} \varepsilon_{23} \\
\sqrt{2} \varepsilon_{31} \\
\sqrt{2} \varepsilon_{12}
\end{array}\right]
$$

The matrix form of the compliance tensor is obtained by inverting (4.8):

$$
\left[\begin{array}{c}
\varepsilon_{11} \\
\varepsilon_{22} \\
\varepsilon_{33} \\
\sqrt{2} \varepsilon_{23} \\
\sqrt{2} \varepsilon_{31} \\
\sqrt{2} \varepsilon_{12}
\end{array}\right]=\left[\begin{array}{cccccc}
s_{11} & s_{12} & s_{13} & \sqrt{2} s_{14} & \sqrt{2} s_{15} & \sqrt{2} s_{16} \\
\bullet & s_{22} & s_{23} & \sqrt{2} s_{24} & \sqrt{2} s_{25} & \sqrt{2} s_{26} \\
\bullet & \bullet & s_{33} & \sqrt{2} s_{34} & \sqrt{2} s_{35} & \sqrt{2} s_{36} \\
\bullet & \bullet & \bullet & 2 s_{44} & 2 s_{45} & 2 s_{46} \\
\bullet & \bullet & \bullet & \bullet & 2 s_{55} & 2 s_{56} \\
\bullet & \bullet & \bullet & \bullet & \bullet & 2 s_{66}
\end{array}\right]\left[\begin{array}{c}
\sigma_{11} \\
\sigma_{22} \\
\sigma_{33} \\
\sqrt{2} \sigma_{23} \\
\sqrt{2} \sigma_{31} \\
\sqrt{2} \sigma_{12}
\end{array}\right]
$$

In the isotropic case, $\underset{\approx}{c}$ can be rewritten as follows:

$$
\underset{\approx}{\boldsymbol{c}}=3 k \underset{\approx}{\boldsymbol{J}}+2 \mu \underset{\approx}{\boldsymbol{K}}
$$

with $k$ bulk modulus, $\mu$ shear modulus, $\boldsymbol{J}$ and $\underset{\approx}{\boldsymbol{K}}$ respectively spherical and deviatoric fourth-order tensorial projectors such that,

$$
\underset{\approx}{\boldsymbol{J}}=\frac{1}{3} \delta_{i j} \delta_{k l} \underline{\boldsymbol{e}}_{i} \otimes \underline{\boldsymbol{e}}_{j} \otimes \underline{\boldsymbol{e}}_{k} \otimes \underline{\boldsymbol{e}}_{l}
$$

and

$$
\underset{\approx}{\boldsymbol{K}}=\underset{\approx}{\boldsymbol{I}}-\underset{\approx}{\boldsymbol{J}}
$$

\subsubsection{The Representative Volume Element}

The question of representativity has been a topic of interest in scientific communities for half a century, especially in the field of materials science, micromechanics and microscopy. Indeed, microstructural heterogeneities play a critical role on the macroscopic physical properties of materials. One common way to account for this underlying complexity is resorting to homogenisation techniques. Most homogenisation approaches, including analytical and computational, require the existence of a representative volume element (RVE). Several definitions have been given for the 
RVE over the past 50 years. A review of this topic can be found in [84]. The classical definition of RVE is attributed to [94], who stated that for a given material the RVE is a sample that is structurally typical of the whole microstructure, i.e. containing a sufficient number of heterogeneities for the macroscopic properties to be independent of the boundary values of traction and displacement. Later, [26] emphasised the role of statistical homogeneity, especially in a volume-averaged sense. This also means that the RVE size considered should be larger than a certain microstructural length for which moduli fluctuate. Hashin [88] made a review on analysis of composite materials in which he referred to statistical homogeneity as a practical necessity. Sab [179] considered that the classical RVE definition for a heterogeneous medium holds only if the homogenised properties tend towards those of a similar periodic medium. This entails that the response over an RVE should be independent of boundary conditions (BC). From numerical simulations on VEs of various sizes, [200] concluded that from a practical viewpoint RVE should be as large as possible. Ostoja-Starzewski [150] considers the RVE to be only defined over a periodic unit-cell or a non-periodic cell containing an infinite number of heterogeneities. Drugan and Willis [61] introduced explicitly the idea of minimising the RVE size, meaning that the RVE would be the smallest material volume for which the apparent and effective properties coincide. Besides, it is worth noticing that for a given material the RVE size for physical property A, e.g. thermal conductivity, is a priori different from the RVE size for physical property B, e.g. elastic moduli. Thus, one has to consider an RVE that depends on the specific investigated property.

Many definitions refer to the separation of scales as a necessary condition for the existence of a RVE. This condition is not always met, i.e. with percolating media or materials with microstructural gradient of properties. This separation of scale involves a comparison between different characteristic lengths:

$-d$, size of microstructural heterogeneities;

$-l$, size of the RVE considered;

- $L$, characteristic length of the applied load.

Previous considerations regarding characteristic lengths can be summarized as follow:

$$
d \ll l \ll L
$$

Nevertheless, Inequality (4.13) is a necessary but not sufficient condition for the applicability of homogenisation. As a matter of fact, quasi-uniform loading, i.e.l $l \ll L$, has to be enforced. Let us consider a measurable property, such as a mechanical strain field. The spatial average of its measured value over a finite volume $V$ converges towards the mathematical expectation of its measured value over a series of samples smaller than $V$ (ensemble average). It is the ergodicity hypothesis. Moreover, ergodicity implies that one sample (or realisation) of volume $V$ contains the statistical information necessary for the description of its microstructure. Also, this entails that heterogeneities are small enough in comparison to the RVE size, i.e. $d \ll l$. If and 
only if these two conditions are met $(d \ll l$ and $l \ll L)$, the existence and uniqueness of an equivalent homogeneous medium for both cases of random and periodic materials can be rigorously proven [179]. Homogenisation is therefore possible.

Taking into account these definitions, and assuming ergodicity for the heterogeneous media considered, [114] proposed a method based on a statistical analysis for computing the minimal RVE size for a given physical property $Z(x), \forall x \in V$ and precision in the estimate of effective properties. The computed RVE size was found to be proportional to the integral range [137], which corresponds to a volume of statistical correlation. This statistical approach is presented in Sect. 4.4.

\subsubsection{Averaging Relations}

Let us consider a given volume element (VE) of volume $V$ without voids or rigid inclusions, for the sake of simplicity. For the spatial average over $V$ of a kinematically compatible strain field $\varepsilon_{\sim}^{\prime}$ which is defined as the symmetric part of the gradient of a kinematically admissible displacement field $\underline{\boldsymbol{u}}^{\prime}$ :

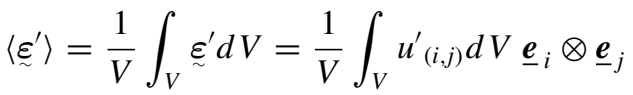

$$
\begin{aligned}
& \left.=\frac{1}{V} \int_{\partial V} u_{(i}^{\prime} n_{j}\right) d S \underline{\boldsymbol{e}}_{i} \otimes \underline{\boldsymbol{e}}_{j} \\
& =\frac{1}{V} \int_{\partial V} \underline{\boldsymbol{u}}^{\prime} \stackrel{s}{\otimes} \underline{\boldsymbol{n}} d S
\end{aligned}
$$

with $\underline{\boldsymbol{u}}^{\prime} \stackrel{s}{\otimes} \underline{\boldsymbol{n}}$ and $u_{(i, j)}^{\prime}$ denoting the symmetric part of the resulting tensor. If one considers now the spatial average of a statically admissible stress field $\boldsymbol{\sigma}^{*}$, i.e. $\boldsymbol{\sigma}^{*} \cdot \boldsymbol{\nabla}=\underline{\mathbf{0}}$ in $V$, it yields:

$$
\begin{aligned}
\left\langle\underline{\boldsymbol{\sigma}}^{*}\right\rangle & =\frac{1}{V} \int_{V} \underline{\sim}^{*} d V=\frac{1}{V} \int_{V} \sigma_{i j}^{*} d V \underline{\boldsymbol{e}}_{i} \otimes \underline{\boldsymbol{e}}_{j} \\
& =\frac{1}{V} \int_{V} \sigma^{*}{ }_{(i k} \delta_{j) k} d V \underline{\boldsymbol{e}}_{i} \otimes \underline{\boldsymbol{e}}_{j} \\
& =\frac{1}{V} \int_{V} \sigma^{*}{ }_{(i k} x_{j), k} d V \underline{\boldsymbol{e}}_{i} \otimes \underline{\boldsymbol{e}}_{j} \\
& \left.=\frac{1}{V} \int_{\partial V} \sigma^{*}{ }_{(i k} n_{k} x_{j}\right) d S \underline{\boldsymbol{e}}_{i} \otimes \underline{\boldsymbol{e}}_{j} \\
& =\frac{1}{V} \int_{\partial V}\left(\boldsymbol{\sigma}^{*} \cdot \underline{\boldsymbol{n}}\right) \stackrel{s}{\otimes} \underline{\boldsymbol{x}} d S
\end{aligned}
$$


From these averaging relations, we can define the elastic strain energy density $\mathcal{E}^{\mathrm{el}}$ such that,

$$
\begin{aligned}
2 \mathcal{E}^{\mathrm{el}} & =\left\langle\boldsymbol{\sigma}^{*}:{\left.\underset{\sim}{\boldsymbol{\varepsilon}^{\prime}}\right\rangle}\right. \\
& =\frac{1}{V} \int_{V}{\underset{\sim}{\boldsymbol{\sigma}}}^{*}:{\underset{\sim}{\boldsymbol{\varepsilon}}}^{\prime} d V \\
& =\frac{1}{V} \int_{V} \sigma_{i j}^{*} u^{\prime}(i, j) \\
& =\frac{1}{V} \int_{V}\left(\sigma^{*}{ }_{i j} u_{i}^{\prime}\right)_{, j} d V \\
& =\frac{1}{V} \int_{\partial V} \sigma^{*}{ }_{i j} n_{j} u_{i}^{\prime} d S \\
& =\frac{1}{V} \int_{\partial V}\left(\underline{\boldsymbol{\sigma}}^{*} \cdot \underline{\boldsymbol{n}}\right) \cdot \underline{\boldsymbol{u}}^{\prime} d S
\end{aligned}
$$

\subsubsection{Boundary Conditions}

It is necessary to set boundary conditions to the volume $V$ considered in order to solve the constitutive equations in the case of statics. Let us consider three types of boundary conditions.

\subsubsection{Kinematic Uniform Boundary Conditions-KUBC}

Displacement $\underline{\boldsymbol{u}}$ is prescribed for any material point $\underline{\boldsymbol{x}}$ on the boundary $\partial V$ such that,

$$
\underline{\boldsymbol{u}}=\underset{\sim}{\boldsymbol{E}} \cdot \underline{\boldsymbol{x}} \quad \forall \underline{\boldsymbol{x}} \in \partial V
$$

with $\underset{\sim}{\boldsymbol{E}}$ second-order macroscopic strain tensor, which is symmetric and independent of $\underline{\boldsymbol{x}}$. It follows from (4.17) and (4.14):

$$
\langle\varepsilon\rangle=\frac{1}{V} \int_{V} \underset{\sim}{\varepsilon} d V=\underset{\sim}{\boldsymbol{E}}
$$

The macroscopic stress tensor is then defined as the spatial average of the local stress field:

$$
\underset{\sim}{\boldsymbol{\Sigma}}:=\langle\underset{\sim}{\boldsymbol{\sigma}}\rangle=\frac{1}{V} \int_{V} \underset{\sim}{\boldsymbol{\sigma}} d V
$$




\subsubsection{Static Uniform Boundary Conditions-SUBC}

Traction $\underline{\boldsymbol{t}}$ is prescribed for any material point $\underline{\boldsymbol{x}}$ on $\partial V$ such that,

$$
\underline{\boldsymbol{t}}=\underset{\sim}{\boldsymbol{\Sigma}} \cdot \underline{\boldsymbol{n}} \quad \forall \underline{\boldsymbol{x}} \in \partial V
$$

with $\underset{\sim}{\Sigma}$ second-order macroscopic stress tensor, which is symmetric and independent of $\underline{x}$. It follows from (4.20) and (4.15):

$$
\langle\underset{\sim}{\boldsymbol{\sigma}}\rangle=\frac{1}{V} \int_{V} \underset{\sim}{\boldsymbol{\sigma}} d V=\underset{\sim}{\boldsymbol{\Sigma}}
$$

The macroscopic strain tensor is then defined as the spatial average of the local strain field:

$$
\underset{\sim}{\boldsymbol{E}}:=\langle\underset{\sim}{\varepsilon}\rangle=\frac{1}{V} \int_{V} \underset{\sim}{\varepsilon d V}
$$

\subsubsection{Periodic Boundary Conditions-PBC}

For PBC, the displacement field $\underline{\boldsymbol{u}}$ can be dissociated into a part given by the macroscopic strain tensor $\underset{\sim}{\boldsymbol{E}}$ and a periodic fluctuation field for any material point $\underline{\boldsymbol{x}}$ of $V$, such that:

$$
\underline{\boldsymbol{u}}=\underline{\sim} \cdot \underline{\boldsymbol{x}}+\underline{\boldsymbol{v}} \quad \forall \underline{\boldsymbol{x}} \in V
$$

with $\underline{v}$ the periodic fluctuations vector, i.e. taking the same value on two homologous points $\underline{\boldsymbol{x}}^{+}$and $\underline{\boldsymbol{x}}^{-}$of $\partial V$. Furthermore, the traction vector $\underline{\boldsymbol{t}}=\boldsymbol{\sigma} \cdot \underline{\boldsymbol{n}}$ fulfills antiperiodic conditions such that,

$$
\begin{gathered}
\underline{\boldsymbol{\sigma}}^{+} \cdot \underline{\boldsymbol{n}}^{+}+\underline{\boldsymbol{\sigma}}^{-} \cdot \underline{\boldsymbol{n}}^{-}=\underline{\mathbf{0}} \\
\underline{\boldsymbol{v}}^{+}-\underline{\boldsymbol{v}}^{-}=\underline{\mathbf{0}}
\end{gathered}
$$

Periodicity is denoted by \# while anti-periodicity is denoted by -\#. A dual approach exists; it consists in prescribing a macroscopic stress to the cell. However we do not develop this approach here, cf. [142] for details.

\subsubsection{Hill-Mandel Condition}

Let us consider a volume $V$ with two independent local fields ${\underset{\sim}{\prime}}^{\prime}$ and $\boldsymbol{\sigma}^{*}$ such that $\boldsymbol{\varepsilon}_{\sim}^{\prime}$ is kinematically compatible and $\boldsymbol{\sigma}_{\sim}^{*}$ is statically admissible. If $\boldsymbol{\sigma}^{*}$ verifies SUBC, 


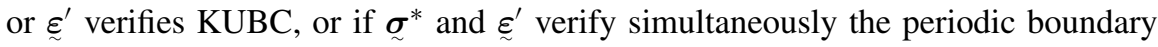
conditions, then:

$$
\left\langle\boldsymbol{\sim}^{*}:{\underset{\sim}{\varepsilon}}^{\prime}\right\rangle=\left\langle\underset{\sim}{\sigma^{*}}\right\rangle:\left\langle\underset{\sim}{\varepsilon^{\prime}}\right\rangle
$$

Thus, one obtains the following equivalence for the three types of boundary conditions:

$$
\langle\underset{\sim}{\boldsymbol{\sigma}}: \underset{\sim}{\boldsymbol{\varepsilon}}\rangle=\langle\underset{\sim}{\boldsymbol{\sigma}}\rangle:\langle\underset{\sim}{\varepsilon}\rangle
$$

which corresponds to the Hill macrohomogeneity condition [95]. This ensures that the mechanical work density at the microscale is preserved while scaling up to the macroscopic level.

\subsubsection{Effective Properties Versus Apparent Properties}

When determining the properties of the volume $V$ smaller than the RVE, apparent properties are considered. The apparent properties converge towards the effective properties once $V \geq V_{\mathrm{RVE}}$.

The micromechanical linear elastic problem admits a unique solution, up to a rigid body displacement for SUBC and a periodic translation for PBC. Let us consider two fourth-order tensors $\underset{\sim}{\boldsymbol{A}}$ and $\underset{\sim}{\boldsymbol{B}}$ accounting respectively for strain localization and stress concentration:

$$
\underset{\sim}{\varepsilon(\underline{x}})=\underset{\approx}{\boldsymbol{A}}(\underline{\boldsymbol{x}}): \underset{\sim}{\boldsymbol{E}} \quad \forall \underline{\boldsymbol{x}} \in V \text { and } \forall \underset{\sim}{\boldsymbol{E}}
$$

and

$$
\underset{\sim}{\boldsymbol{\sigma}}(\underline{\boldsymbol{x}})=\underset{\approx}{\boldsymbol{B}}(\underline{\boldsymbol{x}}): \underset{\sim}{\boldsymbol{\Sigma}} \quad \forall \underline{\boldsymbol{x}} \in V \text { and } \forall \underset{\sim}{\boldsymbol{\Sigma}}
$$

such that,

$$
\langle\underset{\approx}{\boldsymbol{A}}\rangle=\langle\underset{\approx}{\boldsymbol{B}}\rangle=\underset{\approx}{\boldsymbol{I}}
$$

Let us consider the elastic moduli $\underset{\approx}{\boldsymbol{c}}(\underline{\boldsymbol{x}})$ and the compliances $\underset{\approx}{\boldsymbol{s}}(\underline{\boldsymbol{x}})$, then:

$$
\boldsymbol{\sigma}(\underline{\boldsymbol{x}})=\underset{\approx}{\boldsymbol{c}}(\underline{\boldsymbol{x}}): \underline{\sim}_{\boldsymbol{\varepsilon}}(\underline{\boldsymbol{x}}) \quad \forall \underline{\boldsymbol{x}} \in V
$$

and

$$
\underset{\sim}{\varepsilon}(\underline{x})=\underset{\approx}{s}(\underline{x}): \underset{\sim}{\boldsymbol{\sigma}}(\underline{x}) \quad \forall \underline{x} \in V
$$

Thus,

$$
\underset{\sim}{\boldsymbol{\Sigma}}=\langle\underset{\sim}{\boldsymbol{\sigma}}\rangle=\langle\underset{\approx}{\boldsymbol{C}}: \underset{\sim}{\boldsymbol{\varepsilon}}\rangle=\langle\underset{\approx}{\boldsymbol{c}}: \underset{\approx}{\boldsymbol{A}}: \underset{\sim}{\boldsymbol{E}}\rangle=\langle\underset{\approx}{\boldsymbol{c}}: \underset{\approx}{\boldsymbol{A}}\rangle: \underset{\sim}{\boldsymbol{E}}
$$


and

$$
\underset{\sim}{\boldsymbol{E}}=\langle\underset{\sim}{\boldsymbol{\varepsilon}}\rangle=\langle\underset{\approx}{\boldsymbol{S}}: \underset{\sim}{\boldsymbol{\sigma}}\rangle=\langle\underset{\approx}{\boldsymbol{s}}: \underset{\approx}{\boldsymbol{B}}: \underset{\sim}{\boldsymbol{\Sigma}}\rangle=\langle\underset{\approx}{\boldsymbol{s}}: \underset{\sim}{\boldsymbol{B}}\rangle: \underset{\sim}{\boldsymbol{\Sigma}}
$$

We can define ${\underset{\approx}{C}}_{E}^{\text {app }}$ and $\underset{\approx}{\underset{S}{\text { app }}}$, fourth-order symmetric tensors, accounting respectively for the apparent elastic moduli and compliances of the volume $V$ considered such that,

$$
\underset{E}{\boldsymbol{C}} \mathrm{app}=\langle\underset{\approx}{\boldsymbol{c}}: \underset{\approx}{\boldsymbol{A}}\rangle
$$

and

$$
\underset{\Sigma}{\operatorname{Sapp}}=\langle\underset{\approx}{\boldsymbol{S}}: \underset{\approx}{\boldsymbol{B}}\rangle
$$

These equations show that homogenized properties are not usually obtained by a simple rule of mixtures. $\mathcal{E}^{\mathrm{el}}$ :

Also, one can define the apparent properties from the elastic strain energy density

$$
\left.\mathcal{E}^{\mathrm{el}}=\frac{1}{2}\langle\underset{\sim}{\boldsymbol{\sigma}}: \underset{\sim}{\boldsymbol{\varepsilon}}\rangle=\frac{1}{2} \underset{\sim}{\boldsymbol{\varepsilon}}: \underset{\approx}{\boldsymbol{c}}: \underset{\sim}{\boldsymbol{\varepsilon}}\right\rangle=\frac{1}{2} \underset{\sim}{\boldsymbol{E}}:\left\langle\underset{\approx}{\boldsymbol{A}^{T}}: \underset{\approx}{\boldsymbol{c}}: \underset{\approx}{\boldsymbol{A}}\right\rangle: \underset{\sim}{\boldsymbol{E}}
$$

and

$$
\mathcal{E}^{\mathrm{el}}=\frac{1}{2}\langle\underset{\sim}{\boldsymbol{\sigma}}: \underset{\sim}{\boldsymbol{\varepsilon}}\rangle=\frac{1}{2}\langle\underset{\sim}{\boldsymbol{\sigma}}: \underset{\approx}{\boldsymbol{s}}: \underset{\sim}{\boldsymbol{\sigma}}\rangle=\frac{1}{2} \underset{\sim}{\boldsymbol{\Sigma}}:\left\langle\underset{\sim}{\boldsymbol{B}^{T}}: \underset{\sim}{\boldsymbol{s}}: \underset{\sim}{\boldsymbol{B}}\right\rangle: \underset{\sim}{\boldsymbol{\Sigma}}
$$

This way, we obtain a new definition of the apparent elastic moduli and compliances:

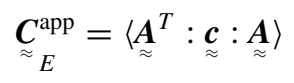

and

$$
\underset{\Sigma}{\operatorname{Sapp}}=\left\langle\underset{\approx}{\boldsymbol{B}}{ }_{\Sigma}^{T}: \underset{\approx}{\boldsymbol{S}}: \underset{\approx}{\boldsymbol{B}}\right\rangle
$$

This new definition justifies the symmetric nature of the apparent elastic moduli and compliance tensors. By applying the Hill-Mandel lemma (cf. Sect. 4.2.5) one can prove the equivalence between direct and energetic definitions [185].

According to [179], for an elementary volume $V$ large enough $\left(V>V_{\mathrm{RVE}}\right)$, the apparent properties do not depend on the boundary conditions and match with the effective properties of the considered material, then:

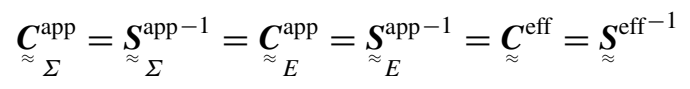

For volumes ( $V \geq V_{\mathrm{RVE}}$ ), based on energetic considerations and the subadditivity property of the effective elastic moduli tensor, [98] proposed the so-called partition theorem. The effective properties can be bounded by the following inequalities: 


$$
\begin{aligned}
& \underset{\Sigma}{\boldsymbol{C}}{ }_{\Sigma}^{\mathrm{app}} \leq{\underset{\approx}{\boldsymbol{C}}}^{\mathrm{eff}} \leq \underset{\approx}{\boldsymbol{C}_{E}^{\mathrm{app}}} \\
& {\underset{\approx}{S}}_{E}^{\mathrm{app}} \leq{\underset{\approx}{S}}_{\mathrm{eff}} \leq{\underset{\sim}{\mathrm{S}}}_{\Sigma}^{\mathrm{app}}
\end{aligned}
$$

These inequalities have to be considered in the sense of quadratic forms. For elementary volumes smaller than the RVE, using the same arguments but for partitions of different sizes, [98] derived hierarchical inequalities regarding apparent and effective properties. Coarse and fine partitions are considered and their respective statistical apparent properties are denoted by indices ${ }_{c}$ and : $_{\text {: }}$

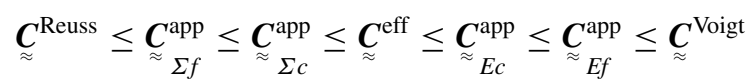

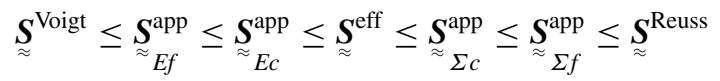

$\underset{\approx}{\boldsymbol{C} \text { Voigt }}, \underset{\approx}{\text { Voigt }}, \underset{\approx}{\boldsymbol{C} \text { Reuss }}$ and $\underset{\approx}{\boldsymbol{S}}{ }^{\text {Reuss }}$ refer to the classical Voigt and Reuss bounds [172, 206]. The inequalities presented above can be used for verification of computational homogenisation results, as it was done for instance in [114, 115] for elastic and thermal properties. Moreover, the bounds $\underset{\approx}{C_{\Sigma}}$ app and $\underset{\approx}{\boldsymbol{C}}$ app are usually far apart when the contrast of properties between phases is large. If the microstructure features a matrix phase, tighter bounds can be obtained by choosing elementary volumes including only the matrix at the boundary, as shown in [182, 183].

\subsubsection{Computational Homogenization Using the Finite Element Method}

In order to determine homogenized mechanical properties for a given microstructure, one has to solve boundary value problems in statics. The finite element (FE) method has proved to be quite an efficient technique to solve this kind of problems even in the case of highly nonlinear phenomena [29, 38, 81].

\subsubsection{FE Formulation of the Principle of Virtual Work}

Galerkin's approach for continuum mechanics is implemented and used with the principle of virtual work. In each of the $n$ elements $e$, knowing the nodal displacements $\left\{u_{e}^{*}\right\}$, one can compute the virtual displacement field $\underline{\boldsymbol{u}}^{*}$ and the strain tensor $\varepsilon$ as follows:

$$
\underline{\boldsymbol{u}}^{*}=[N]\left\{u_{e}^{*}\right\}
$$

and,

$$
\underset{\sim}{\varepsilon}=[B]\left\{u_{e}^{*}\right\}
$$


with $[N]$, the shape function matrix and $[B]$, the matrix of shape function derivatives. Then, for all $\left\{u_{e}^{*}\right\}$ with prescribed body forces $\boldsymbol{f}$ and surface forces $\underline{\boldsymbol{F}}$ :

$$
\sum_{e=1}^{n}\left(\int_{V_{e}} \underset{\sim}{\boldsymbol{\sigma}}\left(\left\{u_{e}^{*}\right\}\right)[B]\left\{u_{e}^{*}\right\} d V\right)=\sum_{e=1}^{n}\left(\int_{V_{e}} \underline{\boldsymbol{f}}[N]\left\{u_{e}^{*}\right\} d V+\int_{\partial V_{e}} \underline{\boldsymbol{F}}[N]\left\{u_{e}^{*}\right\} d S\right)
$$

Thus,

$$
\sum_{e=1}^{n}\left(\left\{\mathcal{F}_{e}^{\text {int }}\right\}-\left\{\mathcal{F}_{e}^{\text {ext }}\right\}\right)\left\{u_{e}^{*}\right\}=0
$$

with $\left\{\mathcal{F}_{e}^{\text {int }}\right\}$ and $\left\{\mathcal{F}_{e}^{\text {ext }}\right\}$ respectively internal and external forces, in each element $e$, such that for the global problem:

$$
\left\{\mathcal{F}^{\text {int }}\right\}=\int_{\Omega}[B]^{T} \underset{\sim}{\sigma}\left(\left\{u_{i}^{*}\right\}\right) d V
$$

and,

$$
\left\{\mathcal{F}^{\mathrm{ext}}\right\}=\int_{\Omega}[N]^{T} \underline{\boldsymbol{f}} d V+\int_{\partial \Omega}[N]^{T} \underline{\boldsymbol{F}} d S
$$

Balance between internal and external forces is achieved with a Newton iterative algorithm using the stiffness matrix $[K]$ :

$$
\begin{aligned}
{[K] } & =\frac{\partial\left\{\mathcal{F}^{\text {int }}\right\}}{\partial\left\{u_{i}^{*}\right\}} \\
& =\int_{\Omega}[B]^{T} \frac{\partial\{\sigma\}}{\partial\{\varepsilon\}} \frac{\partial\{\varepsilon\}}{\partial\left\{u_{i}^{*}\right\}} d V \\
& =\int_{\Omega}[B]^{T} \frac{\partial\{\sigma\}}{\partial\{\varepsilon\}}[B] d V
\end{aligned}
$$

which yields, for linear elastic problems:

$$
[K]=\int_{\Omega}[B]^{T}[c][B] d V
$$

\subsubsection{Application to Linear Elasticity}

In the case of linear elasticity within a volume $V$ fulfilling RVE requirements, one can compute the effective elastic moduli $\underset{\approx}{\boldsymbol{C}}$ or compliances $\underset{\approx}{\boldsymbol{S}}$ using (4.6) by prescribing either the macroscopic strain $\underset{\sim}{\boldsymbol{E}}$ or macroscopic stress $\underset{\sim}{\boldsymbol{\Sigma}}$ : 


$$
\begin{gathered}
{\left[\begin{array}{c}
\Sigma_{11} \\
\Sigma_{22} \\
\Sigma_{33} \\
\Sigma_{23} \\
\Sigma_{31} \\
\Sigma_{12}
\end{array}\right]=\left[\begin{array}{cccccc}
C_{11} & C_{12} & C_{13} & C_{14} & C_{15} & C_{16} \\
- & C_{22} & C_{23} & C_{24} & C_{25} & C_{26} \\
- & - & C_{33} & C_{34} & C_{35} & C_{36} \\
- & - & - & C_{44} & C_{45} & C_{46} \\
- & - & - & - & C_{55} & C_{56} \\
- & - & - & - & - & C_{66}
\end{array}\right]\left[\begin{array}{c}
E_{11} \\
E_{22} \\
E_{33} \\
2 E_{23} \\
2 E_{31} \\
2 E_{12}
\end{array}\right]} \\
{\left[\begin{array}{c}
E_{11} \\
E_{22} \\
E_{33} \\
2 E_{23} \\
2 E_{31} \\
2 E_{12}
\end{array}\right]=\left[\begin{array}{llllll}
S_{11} & S_{12} & S_{13} & S_{14} & S_{15} & S_{16} \\
- & S_{22} & S_{23} & S_{24} & S_{25} & S_{26} \\
- & - & S_{33} & S_{34} & S_{35} & S_{36} \\
- & - & - & S_{44} & S_{45} & S_{46} \\
- & - & - & - & S_{55} & S_{56} \\
- & - & - & - & - & S_{66}
\end{array}\right]\left[\begin{array}{c}
\Sigma_{11} \\
\Sigma_{22} \\
\Sigma_{33} \\
\Sigma_{23} \\
\Sigma_{31} \\
\Sigma_{12}
\end{array}\right]}
\end{gathered}
$$

Linear relations thus appear between macroscopic stress and strain, and can readily be used to build up effective compliance and elastic moduli tensors for a given microstructure. The formalism is similar for any linear property, e.g. thermal conductivity. Such an approach has been successfully implemented for architectured materials in $[31,54,56,58,87,101,104]$.

\subsubsection{The Element DOF Method for Periodic Problems}

In the case of periodic boundary conditions (cf. Sect. 4.2.4.3), there is an alternative to the FE formulation presented in Sect. 4.2.7.1. It consists in adding global DOFs shared by all elements. These DOFs correspond to the macroscopic strain components $E_{i j}$ for displacements $v_{i}$, in addition to classical nodal DOFs. The balance equations can thus be written as follows:

$$
\begin{aligned}
\int_{V} \sigma_{i j} u_{i, j} d V & =\int_{V} \sigma_{i j}\left(E_{i k} x_{k}+v_{i}\right)_{, j} d V \\
& =\int_{V} \sigma_{i j} E_{i j} d V+\int_{V} \sigma_{i j} v_{i, j} d V \\
& =\int_{V} \sigma_{i j} E_{i j} d V+\int_{V}^{\int_{V}\left(\sigma_{i j} v_{i}\right)_{, j} d V} \\
& =\int_{V} \sigma_{i j} E_{i j} d V+\underbrace{\int_{\partial i} \sigma_{i} n_{j} d S}_{\partial V} \\
& =\int_{V} \sigma_{i j} d V E_{i j} \\
& =V \Sigma_{i j} E_{i j} \\
& =R E_{i j} E_{i j}
\end{aligned}
$$


The FE problem left to solve concerns the homogeneous strain tensor $E_{i j}$ and its dual $R E_{i j}$, which corresponds to the macroscopic reaction stress. Prescribing $E_{i j}$ corresponds to the macroscopic strain approach, while prescribing $R E_{i j}$ leads to the macroscopic stress approach. In that way, mixed macroscopic problems, e.g.tension, can be solved with periodic boundary conditions. Implementation of additional degrees of freedom in the FE framework is done as follows:

$$
\{\boldsymbol{\varepsilon}\}=[B]\{u\}+\{\underset{\sim}{\boldsymbol{E}}\}
$$

Also,

$$
\{\varepsilon\}=\left[B^{\prime}\right]\left\{u^{\prime}\right\}
$$

with

$$
\left[B^{\prime}\right]=\left[\begin{array}{llllllll}
1 & & & & & & \\
& 1 & & & & & \\
& & 1 & & & & & \\
& & 1 & & & & \\
& & & 1 & & & \\
& & & & 1 & & \\
& & & & & N^{i} & \\
& & & & & & \ddots
\end{array}\right]
$$

and

$$
\{u\}=\left\{\begin{array}{c}
E 11 \\
E 22 \\
E 33 \\
E 23 \\
E 31 \\
E 12 \\
u^{i} \\
\vdots
\end{array}\right\}
$$

\subsubsection{Case of Application: Periodic Auxetics}

\subsubsection{Auxetics}

Auxetic materials are a type of architectured materials exhibiting a negative Poisson's ratio. They present interesting advantages for both functional and structural applications. In the case of isotropic elasticity, mechanical behavior is described by any couple of parameters among these: Young's modulus $E$, Poisson's ratio $\nu$, 
the bulk modulus $k$ and Lamé's coefficients $\lambda$ and $\mu$ (also referred to as the shear modulus). Poisson's ratio is defined as the ratio of the contraction in the transverse direction to the extension in the longitudinal direction. Material stability requires the tensor of elastic moduli to be positive definite, resulting in a positive Young's modulus $E$ and a Poisson's ratio $\nu$ ranging from -1 , for unshearable materials, and 0.5 for incompressible or rubber-like materials. Most materials naturally present a positive Poisson's ratio, although negative Poisson's ratio materials, or auxetics [69], have been engineered since the mid-1980s with the pioneering works of $[4,93]$ and [124]. This new class of materials has been drawing more and more attention since then $[2,7,23,37,55,56,58,68,80,100,113,119,122,125,144,155,169,178$, 193, 194, 212], as well as their potential applications [15, 28, 46, 67, 135]. Auxetic materials have also been expected to present enhanced acoustic damping [131]; this was shown experimentally in $[43,44]$. The use of auxetics as building blocks for wave-guiding metamaterials has also been investigated in [13, 198]. Moreover, experiments on auxetic foams seem to provide evidence of better resistance to crash compared to conventional cellular materials [188].

Poisson's ratio is defined only for isotropic elastic materials. In the anisotropic case, negative apparent Poisson's ratio can be defined as the opposite of the ratio between transverse and longitudinal strains for a given specific direction. There is no restriction anymore on the values of the apparent Poisson ratio $\nu^{*}$. We call anisotropic auxetics, materials for which negative apparent Poisson's ratio is observed for a sufficiently large range of tensile directions.

\subsubsection{Computational Strategy}

The computational framework used for homogenization has been presented in Sect. 4.2.7. For this application, effective mechanical properties are computed over a unit-cell (defined by its periodicity vectors $\underline{\boldsymbol{v}_{\boldsymbol{i}}}$ ) with periodic boundary conditions (PBC) using FE. Homogenization requires separation between micro and macro scales. In the case of periodic homogenization, the computed effective properties correspond to those of an infinite continuum made of periodic tiles.

Let us consider an elementary volume $V$ including a solid phase $V_{s}$ and porous one $V_{p}$. In the latter, the stress field is extended by setting $\underset{\sim}{\boldsymbol{\sigma}}=\underset{\sim}{\mathbf{0}}$ in $V_{p}$. Practically, the macroscopic strain $\underset{\sim}{\boldsymbol{E}}$ and stress $\underset{\sim}{\boldsymbol{\Sigma}}$ are computed by averaging the local fields $\underset{\sim}{\varepsilon}$ and $\boldsymbol{\sigma}$. Within the porous phase, stresses are assumed to be equal to zero. Using periodic boundary conditions, and applying a homogeneous macroscopic strain field $\underset{\sim}{\boldsymbol{E}}$ (cf. Sect. 4.2.7.3), it yields: 


$$
\begin{aligned}
\underset{\sim}{\boldsymbol{\Sigma}} & =\langle\underset{\sim}{\boldsymbol{\sigma}}\rangle=\frac{1}{V} \int_{V} \underset{\sim}{\boldsymbol{\sigma}} d V \\
& =\frac{1}{V} \int_{V_{s}} \underset{\sim}{\boldsymbol{\sigma}} d V+\underbrace{\frac{1}{V} \int_{V_{p}} \underset{\sim}{\boldsymbol{\sigma}} d V}_{=0} \\
& =\frac{V_{s}}{V} \frac{1}{V_{s}} \int_{V_{s}} \underset{\sim}{\boldsymbol{\sigma}} d V \\
& =V_{V}^{s}\langle\underset{\sim}{\boldsymbol{\sigma}}\rangle_{s}
\end{aligned}
$$

with $V_{V}^{s}$, volume fraction of the solid phase. Let us now consider the case of a prescribed homogeneous macroscopic stress $\underset{\sim}{\boldsymbol{\Sigma}}$ :

$$
\begin{aligned}
\underset{\sim}{\boldsymbol{E}} & =\langle\underset{\sim}{\varepsilon}\rangle=\frac{1}{V} \int_{V} \underset{\sim}{\varepsilon d} V \\
& =\frac{1}{V} \int_{V_{s}} \underset{\sim}{\varepsilon d} V+\frac{1}{V} \int_{V_{p}} \underset{\sim}{\varepsilon d} V \\
& =\frac{V_{s}}{V} \frac{1}{V_{s}} \int_{V_{s}} \underset{\sim}{\varepsilon} d V+\frac{V_{p}}{V} \frac{1}{V_{p}} \int_{V_{p}} \underset{\sim}{\varepsilon d} d V \\
& =V_{V}^{s} \underset{\sim}{\varepsilon\rangle_{s}}+V_{V}^{p}\langle\underset{\sim}{\varepsilon}\rangle_{p}
\end{aligned}
$$

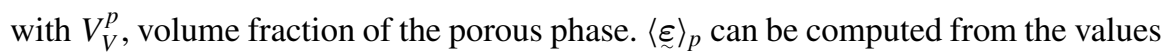
at the boundary of the porous phase.

\subsubsection{Periodic Unit-Cell: Hexachiral Lattice}

This chiral microstructure was first proposed by Lakes [125], then studied in [2, 55, $56,58,169]$. Based on the parameters defined in [2], cell geometry can be described in this way: the circular nodes have radius $r$, the ligaments have length $\mathrm{L}$, and both have in common wall thickness $t$ (cf. Fig. 4.1a) as well as depth d, which in our case is considered infinite due to periodicity conditions along direction 3 . Hence, three dimensionless parameters can be derived as shown in (4.63).

$$
\alpha=\mathrm{L} / \mathrm{r} \quad \beta=\mathrm{t} / \mathrm{r} \quad \gamma=\mathrm{d} / \mathrm{r}
$$

On Fig. 4.1b, $\alpha=5, \beta=0.25$ and $\gamma \rightarrow+\infty$. These parameters correspond to a volume fraction of $15 \%$. The 6 -fold symmetry provides transverse isotropy. 


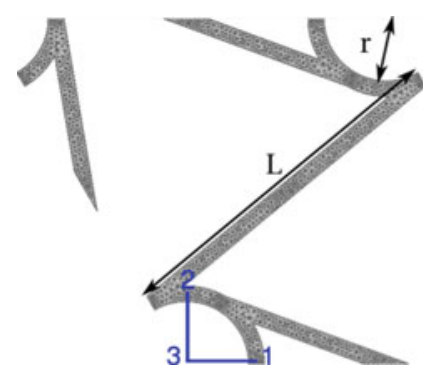

(a) Hexachiral unit-cell

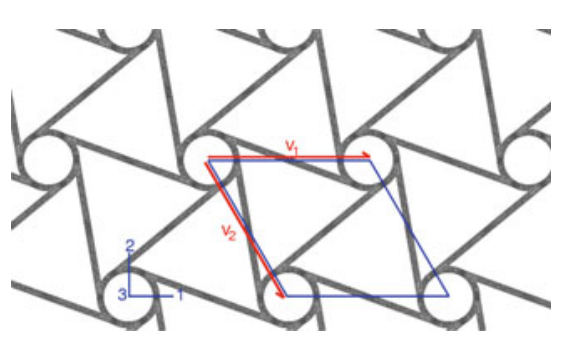

(b) Hexachiral lattice

Fig. 4.1 a Periodic cell with geometric parameters. b Hexachiral lattice with unit-cell (blue) and periodicity vectors $\underline{\boldsymbol{v}_{1}}$ and $\underline{\boldsymbol{v}_{2}}$ (red)

\subsubsection{Effective Elastic Properties}

Elastic moduli tensor $\underset{\sim}{\boldsymbol{C}}$ is computed over a periodic unit-cell using Z-Set FE software. ${ }^{1}$ Meshes are composed of volumic fully-integrated quadratic elements, such as 10-node tetrahedra and 20-node hexahedra, taking into account the finite thickness of the microstructure components. Using the Euler-Bunge [36] angles $\phi, \theta$ and $\psi$ as shown on Fig. 4.2, let us define 3 orthogonal vectors $\underline{\boldsymbol{l}}, \underline{\boldsymbol{m}}$ and $\underline{\boldsymbol{n}}$, such as:

$$
\begin{gathered}
{[\underline{\boldsymbol{l}}]=\left[\begin{array}{c}
\cos (\phi) \cos (\psi)-\sin (\phi) \sin (\psi) \cos (\theta) \\
\sin (\phi) \cos (\psi)+\cos (\phi) \sin (\psi) \cos (\theta) \\
\sin (\psi) \sin (\theta)
\end{array}\right]} \\
{[\underline{\boldsymbol{m}}]=\left[\begin{array}{c}
-\cos (\phi) \sin (\psi)-\sin (\phi) \cos (\psi) \cos (\theta) \\
-\sin (\phi) \sin (\psi)+\cos (\phi) \cos (\psi) \cos (\theta) \\
\cos (\psi) \sin (\theta)
\end{array}\right]} \\
{[\underline{\boldsymbol{n}}]=\left[\begin{array}{c}
\sin (\phi) \sin (\theta) \\
-\cos (\phi) \sin (\theta) \\
\cos (\theta)
\end{array}\right]}
\end{gathered}
$$

Using macroscopic strain and stress tensors $\underset{\sim}{\boldsymbol{E}}(\phi, \theta, \psi)$ and $\underset{\sim}{\boldsymbol{\Sigma}}(\phi, \theta, \psi)$, one can now define the Young modulus $E(\underline{\boldsymbol{l}})$ and effective Poisson ratio $\nu^{*}(\underline{\boldsymbol{l}}, \underline{\boldsymbol{m}})$ for tension along direction $\underline{l}$ :

$$
\begin{gathered}
E=\frac{\underline{\boldsymbol{l}} \cdot \underset{\sim}{\boldsymbol{\Sigma}} \cdot \underline{\boldsymbol{l}}}{\underline{\boldsymbol{l}} \cdot \underset{\sim}{\boldsymbol{E}} \cdot \underline{\boldsymbol{l}}} \\
\nu^{*}=-\frac{\underline{\boldsymbol{m}} \cdot \underset{\sim}{\boldsymbol{E}} \cdot \underline{\boldsymbol{m}}}{\underline{\boldsymbol{l}} \cdot \underline{\sim} \cdot \underline{\boldsymbol{l}}}
\end{gathered}
$$

\footnotetext{
${ }^{1}$ http://www.zset-software.com/.
} 
Fig. 4.2 Euler-Bunge angles

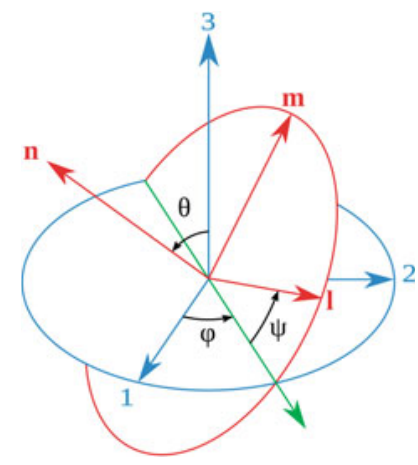

If we now consider simple shear in the plane $(\underline{\boldsymbol{l}}, \underline{\boldsymbol{m}})$, the shear modulus $\mu(\underline{\boldsymbol{l}}, \underline{\boldsymbol{m}})$ can be defined as follows:

$$
\mu=\frac{\underline{\boldsymbol{l}} \cdot \underline{\sim} \cdot \underline{\boldsymbol{m}}}{\underline{\boldsymbol{l}} \cdot \underset{\sim}{\boldsymbol{E}} \cdot \underline{\boldsymbol{m}}}
$$

For $\theta=\psi=0$, elastic moduli and Poisson's ratio are obtained in the plane $(1,2)$ of the microstructure as functions of $\phi$, we will refer to those as in-plane elastic properties. On the other hand, when $\phi=0$ and $\theta=\frac{\pi}{2}$, one obtains moduli and Poisson's ratio within plane $(1,3)$ as functions of $\psi$. These values will be considered as out-of-plane elastic properties.

For comparison purposes, normalized elastic moduli are defined using $f_{V}$, volume fraction of material, local constitutive isotropic elastic material parameters such as $E_{0}$ (Young's modulus) and $\mu_{0}$. Shear modulus $\mu_{0}$ is defined from $E_{0}$ and Poisson's ratio $\nu_{0}$ as follows:

$$
\mu_{0}=\frac{E_{0}}{2\left(1+\nu_{0}\right)} .
$$

Thus, normalized Young's modulus $E^{*}$ is obtained as follows:

$$
E^{*}=\frac{1}{E_{0} f_{V}} E
$$

Normalized shear modulus $\mu^{*}$ is defined in this way:

$$
\mu^{*}=\frac{1}{\mu_{0} f_{V}} \mu
$$

In-plane elastic properties are shown in Table 4.1. The use of auxetic lattices in engineering applications might involve out-of-plane loading. Hence, $\nu^{*}, E^{*}$ and $\mu^{*}$ were plotted against $\psi$ in Fig. $4.3 \mathrm{a}$ (polar plot). For this work, $E_{0}=210,000 \mathrm{MPa}$ and $\nu_{0}=0.3$ are the isotropic elastic properties of the constitutive material. Resulting elastic moduli tensors are presented hereafter as (4.73). Components are expressed in MPa. 
Table 4.1 In-plane Poisson's ratio and normalized elastic moduli

\begin{tabular}{l|l}
\hline & Hexachiral \\
\hline$\nu^{*}$ & -0.74 \\
\hline$E^{*}$ & $2.3 \times 10^{-2}$ \\
\hline$\mu^{*}$ & $2.3 \times 10^{-1}$ \\
\hline
\end{tabular}

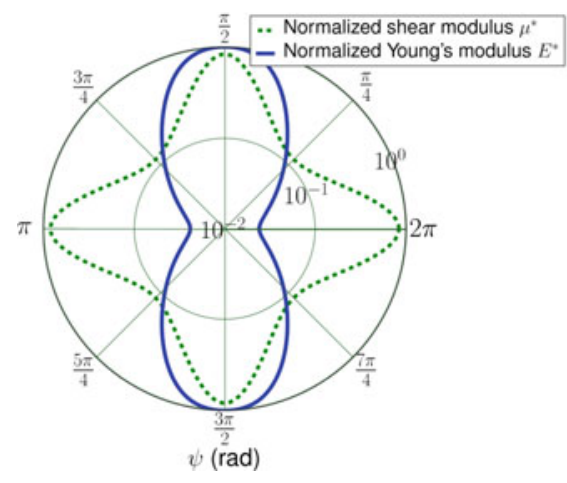

(a) $\mu^{*}, E^{*}=f(\psi)$

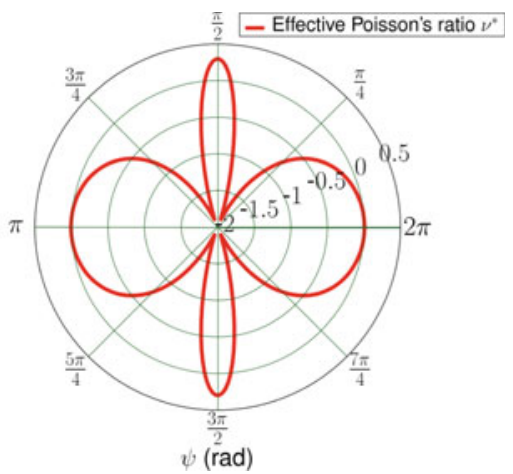

(b) $\nu^{*}=f(\psi)$

Fig. 4.3 Results for hexachiral lattice $\left(\theta=\frac{\pi}{2}, \phi=0\right)$

\subsubsection{Results}

Elastic moduli tensor was computed for the hexachiral lattice as shown in (4.73). Transverse isotropy is verified since $\frac{C_{11}-C_{12}}{2}=C_{66}$.

$$
[\underset{\approx}{\boldsymbol{C}}]=\left[\begin{array}{cccccc}
1650 & -1218 & 130 & 0 & 0 & 0 \\
-1218 & 1650 & 130 & 0 & 0 & 0 \\
130 & 130 & 31968 & 0 & 0 & 0 \\
0 & 0 & 0 & 5075 & 0 & 0 \\
0 & 0 & 0 & 0 & 5075 & 0 \\
0 & 0 & 0 & 0 & 0 & 1434
\end{array}\right]
$$

Components were used to obtain the in-plane properties gathered in Table 4.1. $\nu^{*}$ is underestimated compared to the value from [2], while our estimation of the normalized Young modulus $E^{*}$ is higher. This is discussed later. Figure 4.3a shows an increase of $E^{*}$ when the material is streched out-of-plane, while reaching its maximum value along direction $3\left(\psi=\frac{\pi}{2}\right.$ or $\left.\psi=\frac{3 \pi}{2}\right)$. Figure $4.3 \mathrm{~b}$ shows that Poisson's ratio $\nu^{*}$ is always negative, except for $\psi=0$ or $\psi=\pi$ where $\nu^{*}$ is close to 0 , and $\psi=\frac{\pi}{2}$ or $\psi=\frac{3 \pi}{2}$ where it takes the constitutive material value 0.3 . Normalized shear modulus $\mu^{*}$ fluctuates in a decade around the in-plane value depending on angle $\psi$. 


\subsubsection{Discussion}

Values obtained for $E^{*}$ (cf. Table 4.1) exceed those from [2]. This is due to the boundary conditions of the FEM problem. With periodicity over displacements and nodal force loading, the prescribed loading in [2] corresponds to a static uniform boundary conditions (SUBC) micromechanical problem, which is known to lead to an underestimation of the elastic moduli [114]. On the other hand, the PBC problem gives exact results for an infinite medium. Both the hexachiral lattice presents a strong anisotropy when loaded out-of-plane (cf. Fig. 4.3a). The hexachiral cell has also been studied in the case of second-gradient elasticity, as this model accounts for chirality, hence opening the way for acoustic cloaking applications [13, 16].

\subsection{Computational Homogenization for Elastoplasticity}

The computational homogenization method developed in Sect. 4.2 for elasticity can be extended for elastoplaticity by considering the elastic moduli tensor, as well as an equivalent plasticity model to account for nonlinearities.

A case of application is made from the study of elastoplasticity [55] in the hexachiral auxetic lattice introduced in Sect. 4.2.8.3.

Let us consider the following yield function $f(\underset{\sim}{\sigma})$ :

$$
f(\underset{\sim}{\boldsymbol{\sigma}})=\sigma^{e q}-r
$$

with the von Mises equivalent stress,

$$
\sigma^{e q}=\sqrt{\frac{3}{2} \boldsymbol{\sigma}^{d e v}: \boldsymbol{\sigma}^{d e v}}
$$

where $\sigma_{\sim}^{d e v}$ is the deviatoric part of the stress tensor. Linear isotropic hardening rule is adopted:

$$
r=r_{o}+h p
$$

where $r_{o}$ is the yield stress, $h$ the hardening modulus and $p$ is the cumulative plastic strain variable.

\subsubsection{Plastic Anisotropy}

Local material is now considered isotropic von Mises-elastoplastic. Plastic material parameters are shown in Table 4.2. 
Table 4.2 Local constitutive plastic parameters

\begin{tabular}{l|r}
\hline Yield stress (MPa) & 100 \\
\hline Hardening modulus (MPa) & 1000 \\
\hline
\end{tabular}

Fig. 4.4 Stress (plain curves) and apparent Poisson's ratio (dashed curves) versus strain response for 3 different hardening moduli

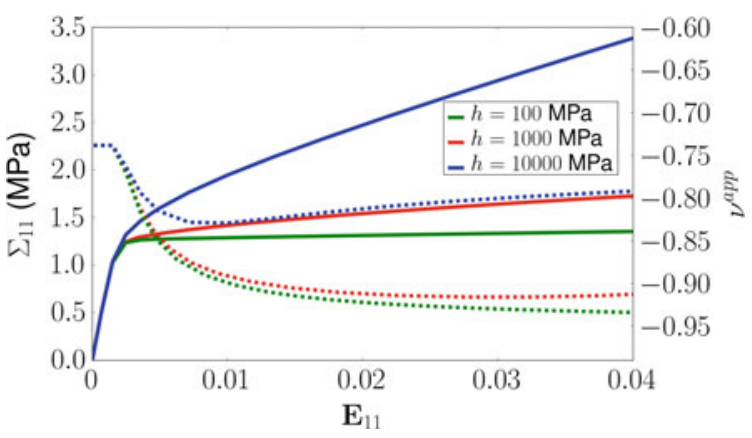

First, the auxetic behavior is investigated. Although the parameters given in Table 4.2 will be used in the following sections, a short parametric study has been performed in order to assess the effect of the hardening modulus on the Poisson ratio. Uniaxial strain-controlled tensile test is performed along direction 1 until 4\% of total macroscopic strain. The homogenized cell exhibits a nonlinear elastoplastic behavior (cf. Fig. 4.4). Now, if one considers the ratio of transverse over longitudinal macroscopic strains, an apparent Poisson ratio can be defined in the nonlinear regime as defined in (4.77) and plotted on Fig. 4.4. From these curves we observe that the auxetic nature of the lattice is kept with plasticity.

The effect is even stronger than in elasticity when the hardening modulus is in the range ( $h=100 \mathrm{MPa}$ and $h=1000 \mathrm{MPa}$ ). The auxetic effect is dependent on the size of the plastic zone in the unit cell. If the plastic zone is confined in a small domain around the junction between the rotating nodes and the connecting beams, as shown on Fig. 4.5 for low values of the hardening modulus, the auxetic deformation mechanism is strengthened. For $h=10,000 \mathrm{MPa}$, the plastic zone spreads almost over the entire cell, thus fading the effect of plasticity on the auxetic behavior. The hardening modulus value, $h=1000 \mathrm{MPa}$, is kept for the rest of this work since it is of the same order of magnitude of several common alloys.

$$
\nu^{\mathrm{app}}=-\frac{\mathbf{E}_{22}}{\mathbf{E}_{11}}
$$

Now, anisotropy in the plastic regime is investigated. As a matter of fact, there is no guarantee for the 6-fold symmetric material to behave isotropically in the plastic regime. Polar plots shown on Fig. 4.6a-c are obtained from uniaxial tensile and shear tests in every direction of the plane $(1,2)$. Each point corresponds to a test for a different direction with angle $\phi$ from the principal direction 1 of the structure defined 


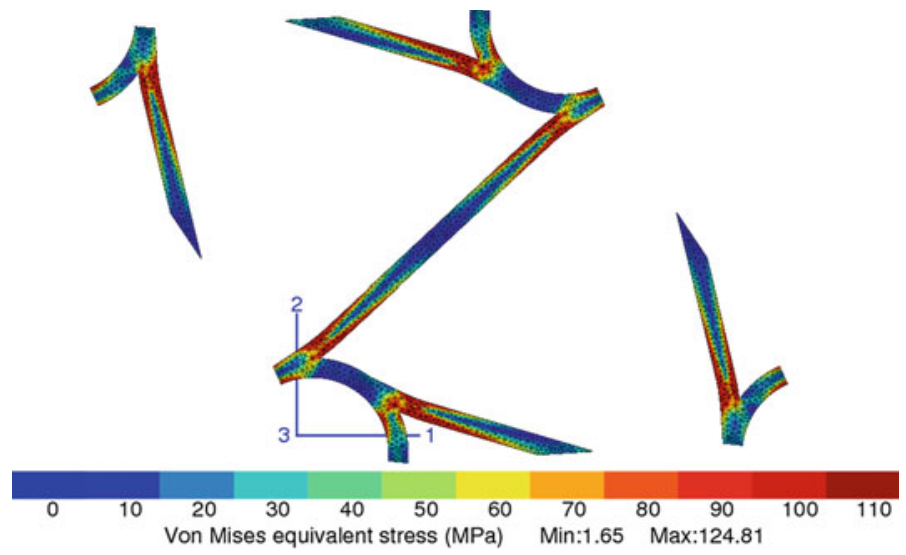

Fig. 4.5 Deformed shape of a periodic unit-cell after $4 \%$ of total strain, with von Mises equivalent stress map $(h=1000 \mathrm{MPa})$

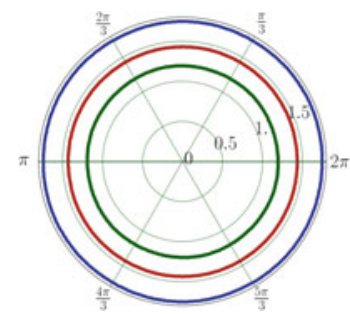

(a) Tensile stress (MPa)

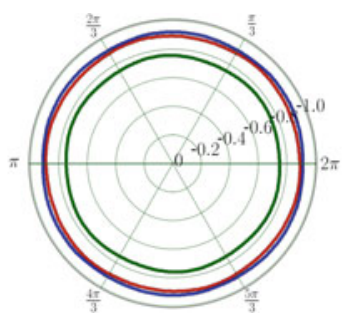

(b) Poisson's ratio

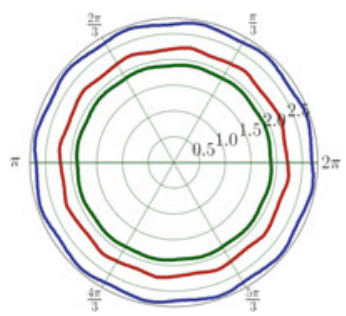

(c) Shear stress (MPa)

Fig. 4.6 a Tensile stress, $\mathbf{b}$ apparent Poisson's ratio, and $\mathbf{c}$ shear stress for $0.2 \%$ (green), $1 \%$ (red) and $4 \%$ (blue) total strain

on Fig. 4.5. Figure 4.6a, c shows stress level versus angle $\phi$ for three given strain states: respectively $0.2 \%$ (green), $1 \%$ (red) and $4 \%$ (blue) total strain for tension, and $0.1,0.5$ and $2 \%$ for shear. Figure $4.6 \mathrm{~b}$ uses the same color code but for the apparent Poisson ratio versus angle $\phi$ for the same given tension states. The three plots show a quasi-transversely isotropic response for the hexachiral lattice with plasticity.

\subsubsection{Macroscopic Modeling}

An additional upscaling can be performed. The mesoscopic elastoplastic behavior is now modelled as a constitutive behavior for further use in large structural computations. First, let us consider an isotropic compressible plasticity model such as those developed by Abouaf et al. [1] and Green [85] for porous metals, and by Deshpande 
and Fleck [52] and Miller [143] for cellular materials. An extension to the anisotropic case was proposed by Badiche et al. [17] and Forest et al. [76].

Let us now consider a yield function $f(\underset{\sim}{\boldsymbol{\Sigma}})$ such as,

$$
f(\underset{\sim}{\Sigma})=\Sigma_{e q}-R
$$

where $R$ is the macroscopic yield stress. Moreover, let us adopt the following equivalent yield stress:

$$
\Sigma_{e q}=\sqrt{\frac{3}{2} C \underset{\sim}{\Sigma^{d e v}}:{\underset{\sim}{\Sigma}}^{d e v}+F(\operatorname{Tr} \underset{\sim}{\Sigma})^{2}}
$$

where $\operatorname{Tr} \underset{\sim}{\Sigma}$ is the trace of the stress tensor. $C$ and $F$ are coefficients accounting for the relative influence of deviatoric and hydrostatic stress, they are usually expressed as functions of the porosity $\rho$ for isotropic materials.

Associated plasticity is assumed, such as the macroscopic plastic strain rate is:

$$
\underset{\sim}{\dot{\mathbf{E}}^{p}}=\frac{\partial f}{\partial \underset{\sim}{\boldsymbol{\Sigma}}}=\frac{\dot{p}}{\sigma_{e q}}\left(\frac{3}{2} C{\underset{\sim}{\boldsymbol{\Sigma}}}^{d e v}+F(\operatorname{Tr} \underset{\sim}{\boldsymbol{\Sigma}}) \underset{\sim}{\mathbf{1}}\right)
$$

In the case of uniaxial tension, we define the in-plane plastic Poisson ratio:

$$
\nu^{p}=-\frac{\dot{\mathbf{E}}_{22}^{p}}{\dot{\mathbf{E}}_{11}^{p}}=-\frac{F-\frac{C}{2}}{C+F}=\frac{\frac{C}{2}-F}{C+F}
$$

When $F=0$, incompressible plasticity is recovered. If $C=1$, then $\nu^{p}<0$ for $F>\frac{1}{2}$ and $\lim _{F \rightarrow+\infty} \nu^{p}=-1 . \nu^{p}$ as a function of $F$ is plotted on Fig. 4.7.

Such a plasticity model is not fully capable of describing the anisotropic behavior of our microstructure along direction 3, especially transverse contraction when tension is applied in plane $(1,2)$. In order to simplify the model, instead of using a fully anisotropic Hill tensor applied to the deviatoric stress tensor and a separate contri-

Fig. 4.7 Plastic Poisson's ratio for an isotropic material as a function of parameter $F$, with $C=1$

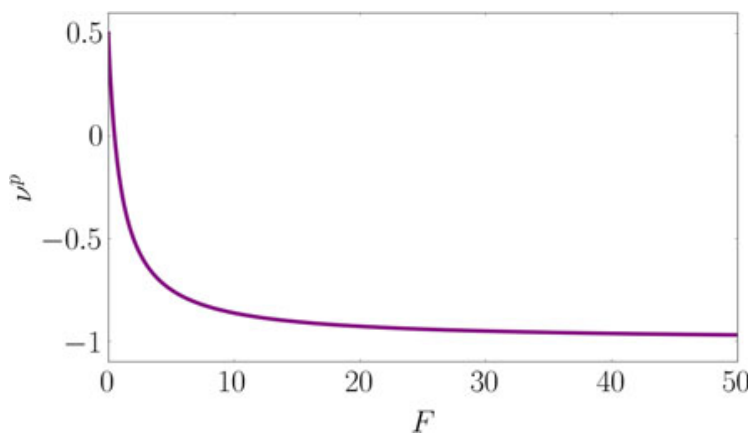


bution of the hydrostatic stress, we consider here a generalized Hill tensor applied to the Cauchy stress tensor.

We consider the same yield function $f(\underset{\sim}{\boldsymbol{\Sigma}})$ as in (4.78) with the following equivalent yield stress:

$$
\Sigma^{e q}=\sqrt{\underset{\sim}{\boldsymbol{\Sigma}: \boldsymbol{H}: \boldsymbol{\Sigma}}}
$$

where $\boldsymbol{H}$ is the applied generalized Hill fourth-rank tensor.

For the hardening rule, we consider an isotropic hardening function with a nonlinear potential and a linear part:

$$
\mathrm{R}=\mathrm{R}_{0}+\mathrm{H} p+\mathrm{Q}\left(1-e^{-\mathrm{b} p}\right) .
$$

\subsubsection{Simulation and Identification}

In order to determine parameters for the model, some of them are determined from reference curves obtained by periodic simulations of the unit-cell. Then comparison between reference data with results computed on a RVE is made and optimization of macroscopic material parameters is run using a simplex algorithm. The experimental database includes tensile, shear and Poisson's ratio curves. While loading in tension, we consider out-of-plane contraction. However, direction 3 and out-of-plane shear are not taken into account. Tensorial components of $\underset{\approx}{\boldsymbol{H}}$ (cf. 4.85) and parameters for the hardening rule (4.83) are thus identified:

$$
R=1.29+8.61 p+0.1\left(1-e^{-140 p}\right)
$$

Comparison between curves from full-field simulations and the identified macroscopic model provides a good correlation as shown on the tensile stress and apparent Poisson's ratio versus strain curve (cf. Fig. 4.8), the shear stress versus strain curve (cf. Fig. 4.9) and the transverse strain vs. longitudinal strain curve (cf. Fig. 4.10).

$$
[\boldsymbol{H}]=\left[\begin{array}{cccccc}
1.00 & 0.9294 & -0.00031 & 0.0006 & 0 & 0 \\
0.9294 & 0.99 & -0.00027 & -0.00067 & 0 & 0 \\
-0.00031 & -0.00027 & \times & 0 & 0 & 0 \\
0.0006 & -0.00067 & 0 & 0.11554 & 0 & 0 \\
0 & 0 & 0 & 0 & \times & 0 \\
0 & 0 & 0 & 0 & 0 & \times
\end{array}\right]
$$


Fig. 4.8 Stress and apparent Poisson's ratio versus strain for full-field simulation and macroscopic model for an uniaxial tensile test along direction 1

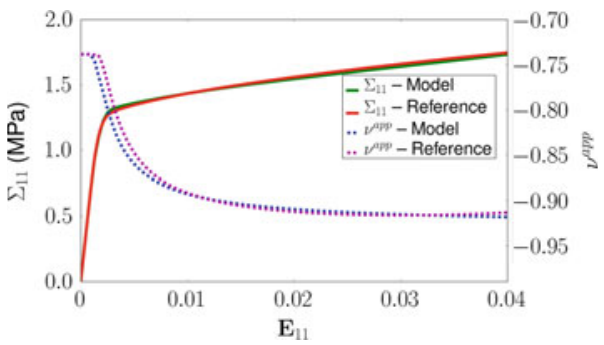

Fig. 4.9 Stress versus strain for full-field simulation and macroscopic model for an pure shear test in plane $(1,2)$

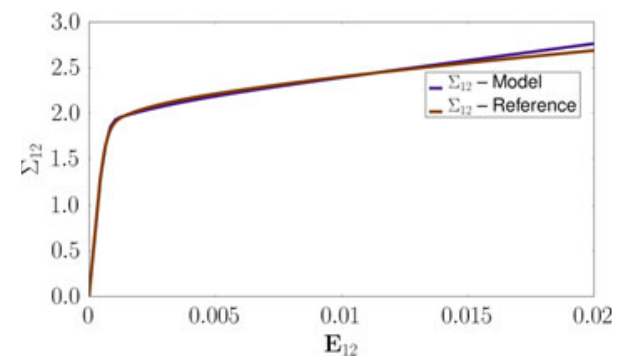

Fig. 4.10 Transverse strain versus longitudinal strain for full-field simulation and macroscopic model for an uniaxial tensile test along direction 1

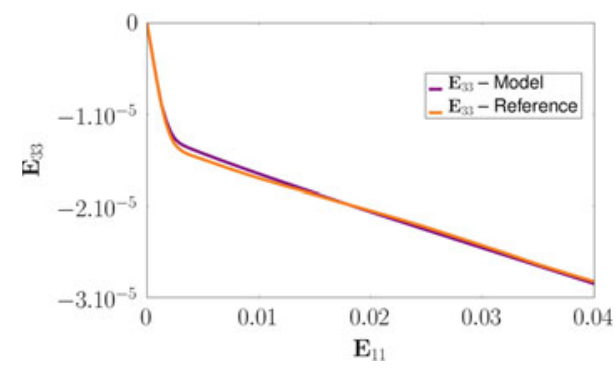

\subsubsection{Conclusions}

Full-field simulations and macroscopic modelling using an anisotropic compressible plasticity framework have been performed for an auxetic microstructure: the hexachiral lattice. Plasticity of auxetics has been explored, showing that the auxetic effect persists and becomes even stronger with plastic yielding. It was also shown that the effect of plasticity on auxeticity fades with the expansion of the plastic zone. The plastic anisotropy for this 6-fold symmetric lattice is becoming weaker with plastic saturation. The proposed fully anisotropic Hill criterion seems to be suitable for modeling periodic architectured cellular materials as it was able to catch negative Poisson's ratio, transverse contraction, and volume change. 


\subsection{Statistical Representative Volume Element Size for Computational Homogenization}

For non-periodic architectured materials, the problem of representativity of samples can be addressed by means of a probabilistic approach giving size-dependent intervals of confidence, which is a well-known approach used in geostatistics [137]. The approach presented [114] is based on the scaling effect on the variance of effective properties in simulations of random media. Several assumptions have to be considered regarding the statistics of the microstructures considered.

\section{Ergodicity hypothesis}

The ergodicity hypothesis is fulfilled for a property or a random function $Z$ when the statistical properties of its measured value (mathematical expectation, variance, etc.) over a finite volume $V$ (spatial average) converge to those estimated over series of independent samples smaller than $V$ (ensemble average), when the volume $V$ goes to infinity. Ergodicity implies that one realization of a volume $V \geq V_{\mathrm{RVE}}$ contains all the statistical information necessary to the description of its microstructure.

\section{Stationarity hypothesis}

The stationarity hypothesis is assumed for a property or a random function $Z$ when its mathematical expectation is constant with respect to time and space.

\section{Statistical homogeneity hypothesis}

A random structure is considered statistically homogeneous, when it is stationary, which means that its probabilistic properties are invariant by translation.

\subsubsection{RVE Size Determination for Media with Finite Integral Range}

Let us consider a microstructure that fulfills the ergodicity and stationarity conditions for a given physical quantity $Z(x)$ regarded as a random function with average $E\{Z(x)\}$ and point variance $D_{Z}^{2}$. The ensemble variance $D_{Z}^{2}(V)$ of its average value $\bar{Z}(V)$ over the domain $\Omega$ with volume $V$ can be obtained using the centered secondorder correlation function $\bar{W}_{2}$ in this way:

$$
D_{Z}^{2}(V)=\frac{1}{V} \iint_{\Omega} \bar{W}_{2}(x-y) d x d y
$$

with

$$
\bar{W}_{2}(h)=E\{(Z(x)-\bar{Z})(Z(x+h)-\bar{Z})\}
$$


For determining the RVE size for the physical property $Z$ one can rely on the geostatistical notion of integral range [39, 109, 112, 126, 127, 138]. The integral range $A_{n}$ is homogeneous to a volume of dimension $n$ in $\mathbb{R}^{n}$. For $n=3$, the integral range is given by:

$$
A_{3}=\frac{1}{D_{Z}^{2}} \int_{\mathbb{R}^{3}} \bar{W}_{2}(h) d h
$$

The physical interpretation of the integral range is such that for a given volume $V$, one can define $n=\frac{V}{A_{3}}$ volume elements for which the $i$ average values $Z_{i}\left(V^{\prime}\right)$ over the $n$ sub-volumes $V^{\prime}=\frac{V}{n}$ are uncorrelated random variables. Hence, for a large specimen, i.e. $V \gg A_{3}$, (4.86) can be rewritten introducing the point variance of $Z$, $D_{Z}^{2}$ as follows:

$$
D_{Z}^{2}(V)=D_{Z}^{2} \frac{A_{3}}{V}
$$

Let us analyze this asymptotic relation. First, in general one has no guarantee on the finiteness of point variance $D_{Z}^{2}$ [137]: let us consider a large domain $\Omega$ and a smaller domain $V \subset \Omega$ that is attainable by means of experimentation or computation, one can then compute an experimental variance which is in fact a function of $\Omega$ supported by $V$, that will increase with $\Omega$. If the variance over $V$ is finite, it should be regarded as a limit of the experimental variance for $\Omega \rightarrow+\infty$. $D_{Z}^{2}$ can be computed over $V$ as follows:

$$
\begin{aligned}
D_{Z}^{2} & =\frac{1}{V} \int_{V}(Z(x)-\bar{Z})^{2} d V \\
& =\frac{1}{V} \int_{V} Z^{2}(x)-\bar{Z}^{2} d V \\
& =\frac{1}{V} \int_{V} Z^{2}(x) d V-\left(\frac{1}{V} \int_{V} Z(x) d V\right)^{2}
\end{aligned}
$$

On the other hand, the ensemble variance $D_{Z}^{2}\left(V^{\prime}\right)$ is computed from the average values $\overline{Z_{i}}$ over $n$ sub-volumes:

$$
\begin{aligned}
D_{Z}^{2}\left(V^{\prime}\right) & =\frac{1}{n} \sum_{i=1}^{n}\left(Z_{i}\left(V^{\prime}\right)-\overline{Z_{i}}\right)^{2} \\
& =\frac{1}{n} \sum_{i=1}^{n} Z_{i}^{2}\left(V^{\prime}\right)-\bar{Z}_{i}^{2} \\
& =\frac{1}{n} \sum_{i=1}^{n} Z_{i}^{2}\left(V^{\prime}\right)-\left(\frac{1}{n} \sum_{i=1}^{n} Z_{i}\left(V^{\prime}\right)\right)^{2}
\end{aligned}
$$


(4.91) uses the average value of the average values $\overline{Z_{i}}$ over $n$ sub-volumes $V^{\prime}$, which is expected to converge towards the effective property $Z_{\text {eff }}$ when $V \rightarrow+\infty$. If $Z_{\text {eff }}$ is already known, it might be of interest to use it instead of $\overline{Z_{i}}$ in order to obtain a better estimate.

If $Z(x)$ is the indicator function of the stationary random set $A$, then one can obtain analytically the variance of the local volume fraction as a function of the point variance as follows:

$$
D_{Z}^{2}=p-p^{2}=p(1-p)
$$

with $p$, probability for a point $x$ to belong to the random set $A$, which is equivalent to the volume fraction of $A$ in $V$.

The asymptotic scaling law given in (4.89) can be used for any additive variable $Z$ over the domain $\Omega$. In the case of elastic properties for instance, average stress $\langle\boldsymbol{\sigma}\rangle$ or strain $\langle\underset{\sim}{\boldsymbol{\varepsilon}}\rangle$ fields have to be computed. For determining the RVE size for a given property $Z$, one thus has to know its integral range $A_{3}$. There is no theoretical covariance for mechanical fields. However, there are two ways to estimate it; first by assuming that $\bar{Z}$ is equal to the arithmetic average of properties (rule of mixtures) for a biphasic medium, hence (4.90) yields:

$$
D_{Z}^{2}=p(1-p)\left(Z_{1}-Z_{2}\right)^{2}
$$

with $Z_{1}$ and $Z_{2}$, respectively property $Z$ of phase 1 and 2. $D_{Z}^{2}$ can also be estimated computationally on the largest virtual sample available, in order to minimize boundary layer effects and obtain a converged value. The approach proposed by di Paola [53] consists in taking only into account the inner part of the simulation volume. This could present an advantage for determining the point variance.

Once the point variance has been estimated for a given property, the integral range can be obtained using the procedure proposed by Matheron [139] for any random function: consider realizations of domains $\Omega$ with an increasing volume $V$ (or non-overlapping sub-domains of large simulations, with a wide range of sizes), the parameter $A_{3}$ can be estimated by fitting the obtained variance according to (4.89):

$$
\log D_{Z}^{2}(V)=\log D_{Z}^{2}+\log A_{3}-\log V
$$

Following the method proposed in [114], itself based on the approach developed in [39], considering a large number $n$ of realisations (or sub-volumes), the following sampling error in the estimation of the effective properties arises:

$$
\epsilon_{\mathrm{abs}}=\frac{2 D_{Z}(V)}{\sqrt{n}}
$$

From which the relative error $\epsilon_{\text {rel }}$ can be defined:

$$
\epsilon_{\mathrm{rel}}=\frac{\epsilon_{\mathrm{abs}}}{\bar{Z}}=\frac{2 D_{Z}(V)}{\bar{Z} \sqrt{n}} \Rightarrow \epsilon_{\mathrm{rel}}^{2}=\frac{4 D_{Z}^{2} A_{3}}{\bar{Z}^{2} n V}
$$


Hence a volume size that we will consider statistically representative can be defined for a prescribed property $Z$, number of realizations $n$ and relative error (e.g. $5 \%$ ):

$$
V_{\mathrm{RVE}}=\frac{4 D_{Z}^{2} A_{3}}{\epsilon_{\mathrm{rel}}^{2} \bar{Z}^{2} n}
$$

This RVE size then depends on the point variance $D_{Z}^{2}$, integral range $A_{3}$ and mean value $\bar{Z}, 3$ parameters that are estimated from simulations.

\subsubsection{Generalization of the Statistical Approach to Microstructures with Non-finite Integral Range}

The method presented above is now adapted and generalized to the case of media with non-finite integral range, especially Poisson linear varieties and Boolean random models made of Poisson linear varieties, e.g. Poisson fibers, which will be used hereafter for modeling non-woven architectured materials. Since the integral range of linear Poisson varieties is not finite [107], (4.89) does not apply anymore. It was proposed in [126] to use a modified scaling law with exponent $\gamma \neq 1$. The variance can thus be rewritten as follows [110]:

$$
D_{Z}^{2}(V)=D_{Z}^{2}\left(\frac{A_{3}^{*}}{V}\right)^{\gamma}
$$

which yields by linearization,

$$
\log D_{Z}^{2}(V)=\log D_{Z}^{2}+\gamma \log A_{3}^{*}-\gamma \log V
$$

$A_{3}^{*}$ is not the integral of the centered second-order correlation function $\bar{W}_{2}(h)$ anymore, as defined before in (4.88). Nonetheless, it is homogeneous to a volume of material and can readily be used to determine RVE sizes which can then be obtained by updating the previous definition for relative error:

$$
\epsilon_{\mathrm{rel}}=\frac{\epsilon_{\mathrm{abs}}}{\bar{Z}}=\frac{2 D_{Z}(V)}{\bar{Z} \sqrt{n}} \Rightarrow \epsilon_{\mathrm{rel}}^{2}=\frac{4 D_{Z}^{2} A_{3}^{* \gamma}}{\bar{Z}^{2} n V^{\gamma}}
$$

Hence yielding an updated definition of the RVE size:

$$
V_{\mathrm{RVE}}=A_{3}^{*} \sqrt{\frac{4 D_{Z}^{2}}{\epsilon_{\mathrm{rel}}^{2} \bar{Z}^{2} n}}
$$


The generalized integral range $A_{3}^{*}$ and scaling-law exponent $\gamma$ can be estimated from simulations as it was done in $[6,57,114]$. When considering statistical RVE sizes of microstructures with non-finite integral range for other properties than morphological ones, for which there is no information about the theoretical value of the point variance $D_{Z}^{2}$, it may be useful to reformulate (4.98) as follows:

$$
D_{Z}^{2}(V)=K V^{-\gamma}
$$

with $K=D_{Z}^{2} A_{3}^{* \gamma}$, leaving only 2 parameters to identify from the statistical data obtained by simulation. We will adopt this formulation when studying Poisson fibers in Sect. 4.4.3. The method for determining statistical RVE sizes has been studied and used for media with finite integral range in the $[6,31,105,106,114,115,133$, $134,152,156,199]$. This approach is implemented for media with infinite integral range in [57], for the case of Poisson fibers.

In most papers, the authors resorted to periodic boundary conditions (PBC) since [114] showed from computational experiments that mean apparent properties obtained with $\mathrm{PBC}$ converge faster towards the effective properties than with the Dirichlet and Neumann-type BC. Nevertheless, KUBC and SUBC can be useful since they correspond to the Voigt and Reuss bounds in elasticity. They can thus be used for bounding the effective properties of random architectured materials. If the microstructure features a matrix phase, tighter bounds can be obtained by choosing elementary volumes including only the matrix at the boundary, as shown in [182].

\subsubsection{Non-woven Architectured Materials}

A challenging candidate material was imagined for assessing the applicability of such methods to architectured materials: stochastic random networks made of infinite fibers, more specifically Poissonian fibrous networks. The determination of the effective properties of Poisson fibers is not trivial as it is a porous medium with infinite integral range. This pathological morphology could give rise to gigantic RVE sizes, maybe even no RVE at all, i.e. yield non-homogenizability in the sense of [14]. Although infinite fibers do not exist in nature, they can be considered a limit case representative of sintered long-fiber non-woven materials, such as those studied by Mezeix et al. [141]. This random fibrous architectured material can be modeled computationally. The generation of such virtual specimens relies on a tridimensional Poisson point implantation process. Due to the long-range correlation induced by the model of random structures chosen, the size of the representative volume element is a priori unknown. Moreover, periodization of these microstructures is generally impossible, making the periodic homogenization tools ineffective. Very large virtual samples computations have to be performed in order to compute their representative volume element sizes a posteriori based on statistical arguments [114] and finally predict their effective mechanical properties. 
Many studies are available regarding finite-length fibrous media and strongly oriented infinite-fiber media. For instance, [50, 158] dealt with the morphology of 3D long-fiber media randomly oriented in-plane, [192] used a 3D random model of randomly oriented long-fibers for the design of an acoustic absorber, and [20, 21] generated virtual samples of long but finite fibers for modeling the mechanics of entangled materials. None of these studies accounted for the representativity of samples. Oumarou et al. [153] computed RVE sizes for 2D random arrays of fibers, using the statistical method of [114]. The works of [89, 161, 162, 196] on 2D fibrous fractal networks also deal with the representativity and homogenization of such, yet self-similar, fibrous media.

The non-woven material model used hereafter corresponds to a 3D stochastic network composed by randomly oriented and distributed infinitely-long interpenetrating rectilinear fibers. It exhibits an infinite integral range [107], i.e. an infinite morphological correlation length; this medium is non-periodizable without modifying its morphology, thus falling beyond the spectrum of periodic homogenization and the definition of RVE proposed by Sab and Nedjar [181].

\subsubsection{Case of Application: RVE Size of Random Fibrous Media}

The example is taken from [57] where the enquiring reader can find details and a full analysis of the problem of representativity in fibrous random media. The results presented hereafter are based on the equations introduced in Sect. 4.2.8.2 for the homogenization of porous media.

As shown on Fig. 4.11, the elementary volume considered in the computation is the volume $V$ that is composed of two complementary phases $V_{f}$ and $V_{p}$, respectively accounting for the Poisson fibers and the porous phase.

\subsubsection{Mixed Mechanical Boundary Conditions-MBC}

A type of mixed BC, appropriate for porous random media, is presented here, instead of SUBC, in order to compute values that we can compare with those obtained using KUBC. Since only a minimal number of DOFs are prescribed on the boundary $\partial V$, mixed mechanical boundary conditions (MBC) are less constraining, or "softer", than KUBC. They differ from the mixed uniform boundary conditions (MUBC) proposed in $[90,91]$ which are restricted, in general, to tensile loading of a volume element. They also differ from the normal mixed BC (NMBC) proposed by Gelebart et al. [82], since those are applied on the whole boundary $\partial V$, similarly to MUBC. The periodicity compatible mixed uniform boundary conditions (PMUBC) were proposed by Pahr and Zysset [154] as a generalization of MUBC, and implemented by Chateau et al. [42]. The BC proposed here are similar to PMUBC, but simpler 
Fig. 4.11 Example of an elementary volume of Poisson fibers used in the simulation

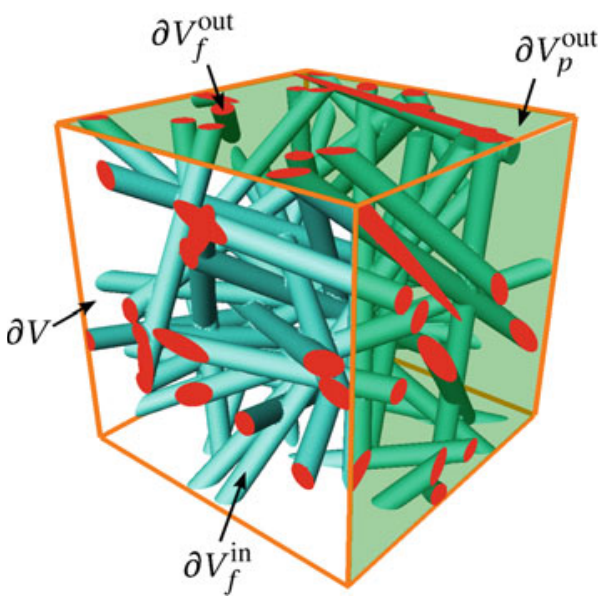

because only 2 loading configurations are considered. MBC are considered with a view to estimating the overall bulk and shear moduli of isotropic random porous media, thus being less general than PMUBC; the 2 loading cases are presented hereafter.

Mixed triaxial loading

Displacement $\underline{\boldsymbol{u}}$ is prescribed along normals on $\partial V$, such that:

$$
\begin{array}{ll}
u_{1}=E_{11} x_{1} & \forall \underline{x} \in F_{1} \\
u_{2}=E_{22} x_{2} & \forall \underline{x} \in F_{2} \\
u_{3}=E_{33} x_{3} & \forall \underline{x} \in F_{3}
\end{array}
$$

The traction vector $\boldsymbol{\sigma} \cdot \underline{\boldsymbol{n}}$ is prescribed in this way:

$$
\begin{array}{ll}
\sigma_{21} n_{1}=\sigma_{31} n_{1}=0 & \forall \underline{x} \in F_{1} \\
\sigma_{12} n_{2}=\sigma_{32} n_{2}=0 & \forall \underline{x} \in F_{2} \\
\sigma_{13} n_{3}=\sigma_{23} n_{3}=0 & \forall \underline{x} \in F_{3}
\end{array}
$$

From (4.62), it yields:

$$
\left\langle\varepsilon_{11}\right\rangle=E_{11} ; \quad\left\langle\varepsilon_{22}\right\rangle=E_{22} ; \quad\left\langle\varepsilon_{33}\right\rangle=E_{33}
$$

The macroscopic stress is computed using (4.61).

\section{Mixed shear loading}

Displacement $\underline{\boldsymbol{u}}$ is prescribed along direction 1 on $F_{2}$ and direction 2 on $F_{1}$, such that: 


$$
\begin{array}{ll}
u_{2}=E_{12} x_{1} & \forall \underline{x} \in F_{1} \\
u_{1}=E_{12} x_{2} & \forall \underline{x} \in F_{2}
\end{array}
$$

Two components of the traction vector $\underset{\sim}{\boldsymbol{\sigma}} \cdot \underline{\boldsymbol{n}}$ are prescribed in this way:

$$
\begin{gathered}
\sigma_{11} n_{1}=\sigma_{31} n_{1}=0 \quad \forall \underline{x} \in F_{1} \\
\sigma_{22} n_{2}=\sigma_{32} n_{2}=0 \quad \forall \underline{x} \in F_{2} .
\end{gathered}
$$

The traction vector is fully prescribed on $F_{3}$ :

$$
\sigma_{33} n_{3}=\sigma_{13} n_{3}=\sigma_{23} n_{3}=0 \quad \forall \underline{x} \in F_{3}
$$

(4.62) gives:

$$
\left\langle\varepsilon_{12}\right\rangle=E_{12}
$$

Details of the calculation can be found in [57]. The boundary value problems for estimating the overall bulk and shear moduli are explicited hereafter for both KUBC and MBC.

\subsubsection{Overall Properties}

The fibers are considered linear elastic following the generalized Hooke law:

$$
\underset{\sim}{\boldsymbol{\sigma}}=\underset{\approx}{c}: \underset{\sim}{\varepsilon} \forall \underline{\boldsymbol{x}} \in V_{f}
$$

with $\underset{\sim}{\boldsymbol{c}}$, fourth-order positive definite tensor of elastic moduli of the isotropic elastic fibers, and $V_{f}$ volume of the fibers.

\section{Overall bulk modulus}

For the case of linear elasticity, $\langle\underset{\sim}{\boldsymbol{\sigma}}: \underset{\sim}{\boldsymbol{\varepsilon}}\rangle$ is used as an estimate of $\left(\underset{\sim}{\boldsymbol{C}}{ }^{\mathrm{eff}}: \underset{\sim}{\boldsymbol{E}}\right): \underset{\sim}{\boldsymbol{E}}$, with $\underset{\approx}{C^{\text {eff }}}$, the tensor of effective elastic moduli of the homogenized medium, that can be rewritten in this way:

$$
{\underset{\approx}{\mathrm{eff}}}^{\mathrm{ef}} 3 k^{\mathrm{eff}} \underset{\approx}{\boldsymbol{J}}+2 \mu^{\mathrm{eff}} \underset{\approx}{\boldsymbol{K}}
$$

with $\underset{\approx}{\boldsymbol{K}}$ and $\boldsymbol{J}$ respectively deviatoric and spherical projector on 2nd-order tensors.

Hence, for a hydrostatic macroscopic strain tensor,

$$
\underset{\sim}{\boldsymbol{E}}=\left[\begin{array}{lll}
1 & 0 & 0 \\
0 & 1 & 0 \\
0 & 0 & 1
\end{array}\right]
$$


it yields:

$$
2 \mathcal{E}^{\mathrm{el}}=\langle\underset{\sim}{\boldsymbol{\sigma}}: \underset{\sim}{\boldsymbol{\varepsilon}}\rangle=\underset{\sim}{\boldsymbol{\Sigma}}: \underset{\sim}{\boldsymbol{E}}=\left(\underset{\sim}{\boldsymbol{e}^{\mathrm{eff}}}: \underset{\sim}{\boldsymbol{E}}\right): \underset{\sim}{\boldsymbol{E}}=3 k^{\mathrm{eff}} \underset{\sim}{\boldsymbol{E}}: \underset{\sim}{\boldsymbol{J}}: \underset{\sim}{\boldsymbol{E}}=9 k^{\mathrm{eff}}
$$

when computed on a given volume element, $k^{\text {app }}=\frac{1}{9}\langle\underset{\sim}{\boldsymbol{\sigma}}: \underset{\sim}{\boldsymbol{\varepsilon}}\rangle$ is an estimate of $k^{\text {eff }}$. Overall shear modulus

For a deviatoric macroscopic strain tensor,

$$
\underset{\sim}{\boldsymbol{E}}=\left[\begin{array}{lll}
0 & \frac{1}{2} & 0 \\
\frac{1}{2} & 0 & 0 \\
0 & 0 & 0
\end{array}\right]
$$

it yields:

$$
2 \mathcal{E}^{\mathrm{el}}=\langle\underset{\sim}{\boldsymbol{\sigma}}: \underset{\sim}{\boldsymbol{\varepsilon}}\rangle=\underset{\sim}{\boldsymbol{\Sigma}}: \underset{\sim}{\boldsymbol{E}}=\left(\underset{\sim}{\boldsymbol{C}}{ }^{\mathrm{eff}}: \underset{\sim}{\boldsymbol{E}}\right): \underset{\sim}{\boldsymbol{E}}=2 \mu^{\mathrm{eff}} \underset{\sim}{\boldsymbol{E}}: \underset{\sim}{\boldsymbol{K}}: \underset{\sim}{\boldsymbol{E}}=\mu^{\mathrm{eff}}
$$

When computed on a given volume element, $\mu^{\text {app }}=\langle\underset{\sim}{\boldsymbol{\sigma}}: \underset{\sim}{\boldsymbol{\varepsilon}}\rangle$ is an estimate of $\mu^{\text {eff }}$.

\subsubsection{Results}

The estimation of elastic properties is now performed on hundreds of realizations. Fibers were attributed the constitutive material properties listed in Table 4.3. The variability of each realization gives rise to different results for apparent elastic properties. Mean values over $n$ realizations of the same size are considered in this section. The number of realizations $n$ fluctuates, depending on the size and BC. Ideally, $n$ should be different for each volume size in order to achieve a similar sampling error for all sizes considered. The fluctuation observed here is due to errors during the meshing step; the larger the virtual sample, the more defects in the geometry are likely to happen. Nevertheless, the values gathered in Table 4.4 are large enough for achieving statistical representativity.

Table 4.3 Constitutive material parameters for Poisson fibers

\begin{tabular}{l|l}
\hline Young's modulus $(\mathrm{GPa})$ & 210 \\
\hline Poisson's ratio & 0.3 \\
\hline Shear modulus $(\mathrm{GPa})$ & 81 \\
\hline Bulk modulus $(\mathrm{GPa})$ & 175 \\
\hline Volume fraction & 0.16 \\
\hline
\end{tabular}


Table 4.4 Number of realizations $n$ considered depending on boundary conditions and simulation size

\begin{tabular}{l|l|l|l|l|l|l|l|l|l|l}
\hline \multirow{2}{*}{ BC } & \multicolumn{2}{|l}{ Volume } \\
\cline { 2 - 13 } & $10^{3}$ & $20^{3}$ & $30^{3}$ & $40^{3}$ & $50^{3}$ & $60^{3}$ & $70^{3}$ & $80^{3}$ & $90^{3}$ & $100^{3}$ \\
\hline$n-$ KUBC $-\overline{k^{\text {app }}}$ & 63 & 63 & 61 & 60 & 56 & 54 & 44 & 51 & 32 & 13 \\
\hline$n-$ KUBC $-\overline{\mu^{\text {app }}}$ & 63 & 63 & 61 & 60 & 54 & 54 & 47 & 50 & 27 & 14 \\
\hline$n-$ MBC $-\overline{k^{\text {app }}}$ & 40 & 60 & 53 & 55 & 45 & 48 & 37 & 46 & 28 & 13 \\
\hline$n-$ MBC $-\overline{\mu^{\text {app }}}$ & 41 & 62 & 58 & 59 & 48 & 49 & 45 & 41 & 11 & 12 \\
\hline
\end{tabular}

Results for the elastic properties are obtained over a large number of realizations (between 11 and 63, depending on their size). For the hydrostatic loading defined in (4.112), the ensemble averaged apparent bulk modulus $\overline{k^{\text {app }}}$ is computed in this way:

$$
\overline{k^{\mathrm{app}}}=\frac{1}{n} \sum_{i=1}^{n}\left(k^{\mathrm{app}}\right)_{i}=\frac{1}{n} \sum_{i=1}^{n}\left(\frac{1}{9}\langle\operatorname{Tr} \sigma\rangle_{f}\right)_{i}
$$

On the other hand, the ensemble averaged apparent shear modulus $\overline{\mu^{\text {app }}}$ is obtained as follows for the shear loading defined in (4.114):

$$
\overline{\mu^{\mathrm{app}}}=\frac{1}{n} \sum_{i=1}^{n}\left(\mu^{\mathrm{app}}\right)_{i}=\frac{1}{n} \sum_{i=1}^{n}\left(\left\langle\sigma_{12}\right\rangle_{f}\right)_{i}
$$

Results for the bulk modulus $\overline{k^{\text {app }}}$ and shear modulus $\overline{\mu^{\text {app }}}$ are shown respectively on Figs. 4.12 and 4.13 for the different volumes of simulation and corresponding number of fibers $(\bar{N})$ considered. The mean values obtained for the largest system size considered $(\bar{N} \simeq 800)$ are given with the corresponding intervals of confidence $\left[\bar{Z} \pm 2 D_{Z}\right]$ in Table 4.5 and compared with analytical bounds. Boundary layer effects are significant for small elementary volume sizes, for both types of BC. Similarly

Fig. 4.12 Mean values for the bulk modulus depending on the number of fibers

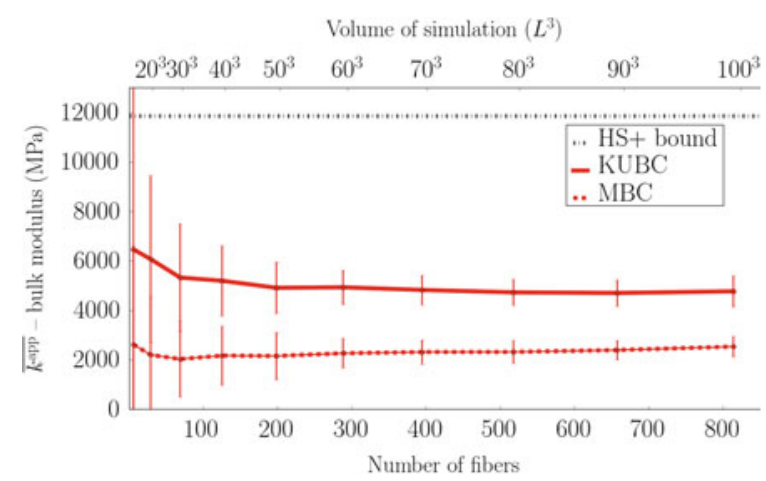


Fig. 4.13 Mean values for the shear modulus depending on the number of fibers

Table 4.5 Bounds and mean values for apparent thermal and elastic properties

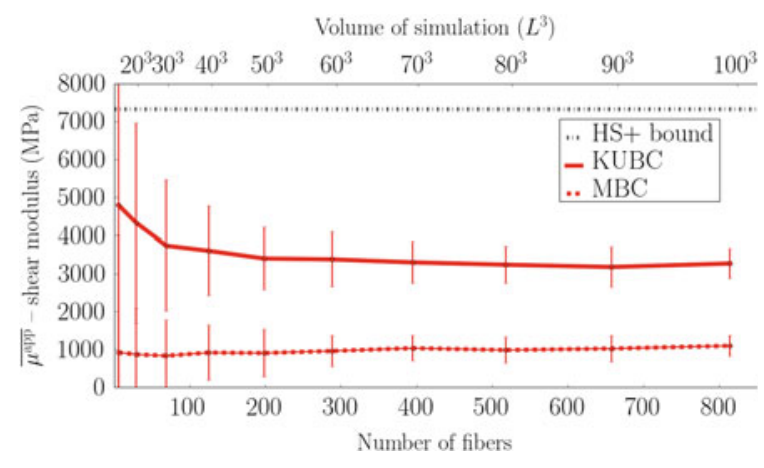

\begin{tabular}{l|l|l}
\hline & $\overline{k^{\mathrm{app}}}(\mathrm{MPa})$ & $\overline{\mu^{\mathrm{app}}}(\mathrm{MPa})$ \\
\hline FE-Uniform & $4763 \pm 624$ & $3270 \pm 394$ \\
\hline FE-Mixed & $2527 \pm 413$ & $1097 \pm 272$ \\
\hline $\begin{array}{l}\text { Hashin-Shtrikman } \\
\text { upper bound }\end{array}$ & 11,839 & 7328 \\
\hline Voigt bound & 28,000 & 12,923 \\
\hline
\end{tabular}

to the results observed for the thermal properties, mean apparent values for KUBC are decreasing with increasing volume size, whereas mean estimates obtained with MBC are slightly increasing with $V$. There are very strong discrepancies for mean values obtained with KUBC and MBC for both bulk and shear moduli, even for the largest volume considered: $\Delta\left(\overline{k^{\mathrm{app}}}\right)=88 \%$ and $\Delta\left(\overline{\mu^{\mathrm{app}}}\right)=198 \%$. Apparent properties obtained for KUBC are constantly above those computed with MBC; this is in accordance with the bounds stated in [91] for mixed boundary conditions, as well as the hierarchies of estimates given by Huet [99].

\subsubsection{Mechanical Fields}

In order to explain the discrepancies observed on the mean apparent properties, let us analyze the mechanical fields coming out of the simulation done on a realization with $V=50^{3}$. For the triaxial loading, mapping of the normalized elastic energy density $\frac{\underset{\sim}{\boldsymbol{\sigma}: \boldsymbol{\sim}^{\boldsymbol{\varepsilon}}}}{V_{f}\left\langle\underset{\sim}{\boldsymbol{\sigma}}: \boldsymbol{\varepsilon}_{f}\right\rangle_{f}}$ is presented on Fig. 4.14 for KUBC and MBC. From this figure, it can be seen that KUBC induce a higher elastic energy density level than with MBC. Also, the strain localization is affecting every fiber for KUBC, while only the favorably oriented fibers are deforming with $\mathrm{MBC}$, resulting in a more homogeneous elastic strain energy density field with KUBC than with MBC. On Fig. 4.15, mapping only $\frac{\varepsilon_{22}}{E_{22}}$, for MBC the localization is clearly confined to both ends of preferentially 
Fig. 4.14 $\frac{\underset{\sigma}{\sigma: \varepsilon}}{V_{f}\langle\boldsymbol{\sigma}: \varepsilon\rangle_{f}}$ mapping for a $V=50^{3}$ realization under hydrostatic load using KUBC (top) and MBC (bottom)

Fig. 4.15 $\frac{\varepsilon_{22}}{E_{22}}$ mapping for a $V=50^{3}$ realization under hydrostatic load using KUBC (top) and MBC (bottom)
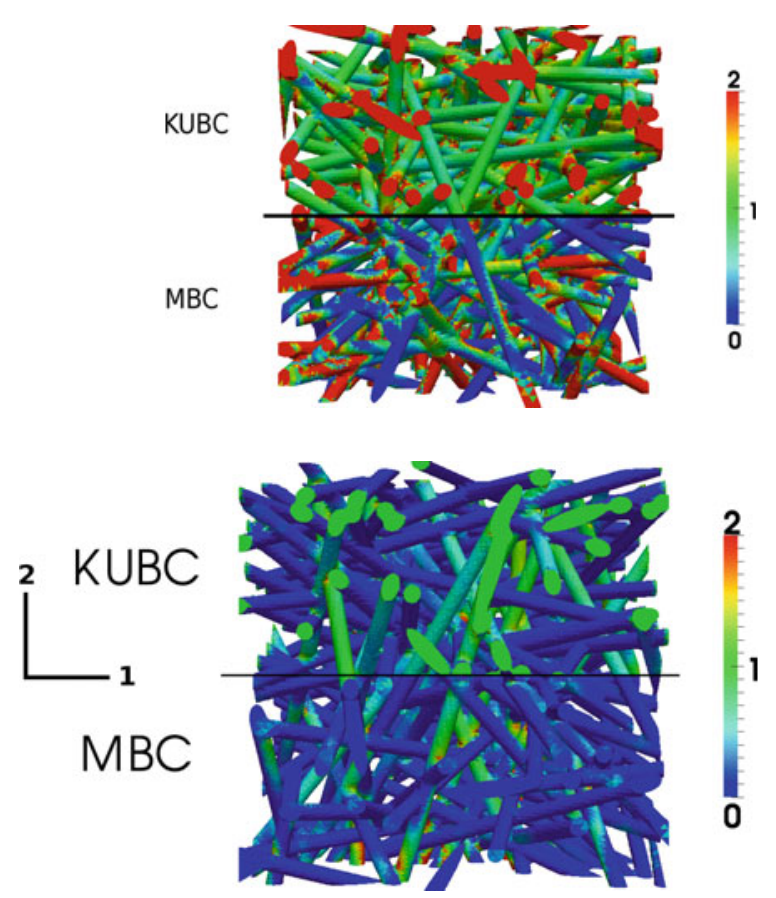

oriented fibers, here along the vertical direction. On the other hand, for KUBC most of the deformation takes place all over the boundary $\partial V$, including fibers that are not preferentially oriented.

\subsubsection{Integral Range, Variance and RVE Size}

The theory introduced in Sect. 4.4.2 is now applied. Using (4.101) and (4.102), it yields:

$$
V_{\mathrm{RVE}}=\left(\frac{4 K}{\epsilon_{\mathrm{rel}}^{2} \bar{Z}^{2} n}\right)^{\frac{1}{\gamma}}
$$

Based on (4.100), the relative error associated with the number of realizations considered (cf. Table 4.4) is computed and presented in Table 4.6. As stated earlier, ideally, the error should be the same for all sample series by adapting the number of realizations. Although the relative error fluctuates, most values are very low, except for the smallest sample sizes.

Variance for the apparent bulk and shear moduli as functions of the volume are shown on Figs. 4.16 and 4.17.

The $\gamma$ exponents of the scaling-law for both moduli were estimated from the results of simulations, by fitting the slope of the variance curves. Only the data 
Table 4.6 Relative error for the samples considered $\epsilon_{\text {rel }}$ depending on boundary conditions and simulation size

\begin{tabular}{l|l|l|l|l|l|l|l|l|l|l}
\hline \multirow{2}{*}{ BC } & \multicolumn{9}{l}{ Volume } \\
\cline { 2 - 12 } & $\begin{array}{l}10^{3} \\
(\%)\end{array}$ & $20^{3}(\%)$ & $\begin{array}{l}30^{3} \\
(\%)\end{array}$ & $\begin{array}{l}40^{3} \\
(\%)\end{array}$ & $\begin{array}{l}50^{3} \\
(\%)\end{array}$ & $\begin{array}{l}60^{3} \\
(\%)\end{array}$ & $\begin{array}{l}70^{3} \\
(\%)\end{array}$ & $\begin{array}{l}80^{3} \\
(\%)\end{array}$ & $\begin{array}{l}90^{3} \\
(\%)\end{array}$ & $\begin{array}{l}100^{3} \\
(\%)\end{array}$ \\
\hline$\epsilon_{\text {rel }}-$ KUBC $-\overline{k^{\text {app }}}$ & 9.9 & 4.9 & 3.7 & 2.9 & 2.5 & 2.1 & 2.0 & 1.7 & 1.9 & 2.6 \\
\hline$\epsilon_{\text {rel }}-$ KUBC $-\overline{\mu^{\text {app }}}$ & 11.9 & 6.2 & 4.7 & 3.7 & 3.3 & 2.7 & 2.6 & 2.2 & 2.7 & 3.3 \\
\hline$\epsilon_{\text {rel }}-$ MBC $-\overline{k^{\text {app }}}$ & 25.6 & 11.7 & 8.8 & 6.0 & 5.3 & 4.1 & 3.9 & 3.0 & 3.3 & 4.2 \\
\hline$\epsilon_{\text {rel }}-\mathrm{MBC}-\overline{\mu^{\text {app }}}$ & 51.6 & 21.8 & 14.7 & 9.8 & 8.8 & 6.8 & 5.6 & 5.4 & 8.9 & 7.1 \\
\hline
\end{tabular}

Fig. 4.16 Variance for the bulk modulus depending on the volume of simulation

Fig. 4.17 Variance for the shear modulus depending on the volume of simulation
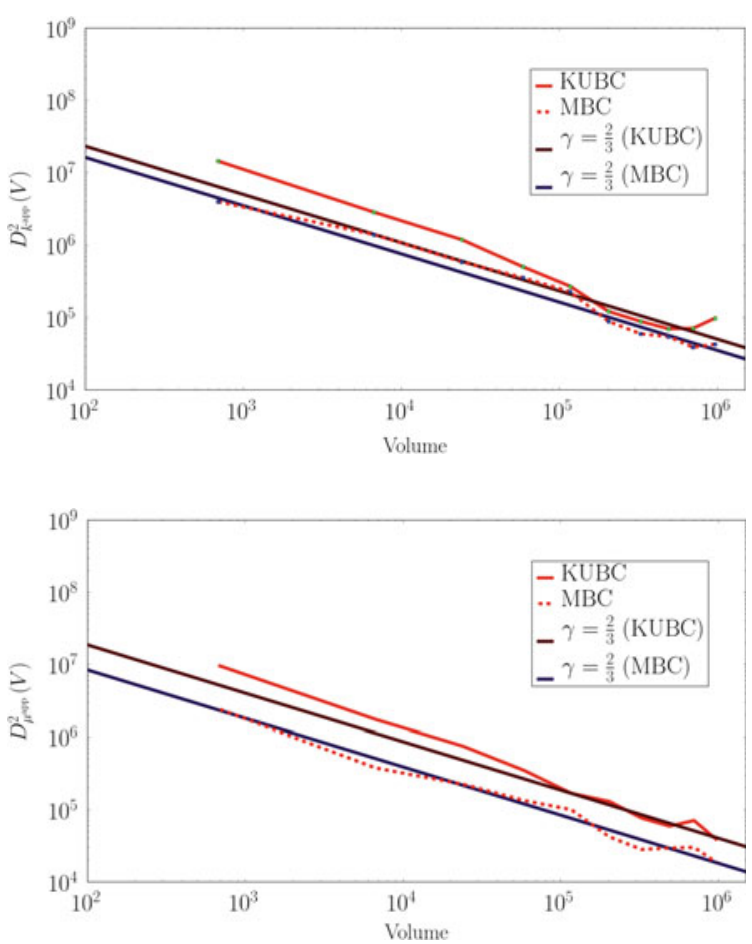

points for volumes $V \geq 40^{3}$ have been considered, in order to mitigate any bias due to boundary layer effects with small volumes. The values obtained in this way are gathered in Table 4.7. For the case of Poisson fibers, [110, 111] gave the theoretical value of $\frac{2}{3}$ for $\gamma$ in (4.98) and (4.102). This value holds only for the indicator function of the Poisson fibers and its mean value, the volume fraction. The value estimated here $(\gamma=0.67)$ verifies the theoretical result of [110]. Results from [6], for 3D randomly distributed long-fibers, show a convergence of the variance on the volume fraction of fibers following a scaling law with exponent $\gamma=0.8$; this value is inbetween the values for infinite fibers $\left(\gamma=\frac{2}{3}\right)$ and short-fibers $(\gamma=1)$, which could be compared to a random distribution of spheres in the extreme case of a shape factor 
Table 4.7 Values for $\gamma$ exponent estimated from the simulation

\begin{tabular}{l|l}
\hline & $\gamma$ \\
\hline$\overline{k^{\mathrm{app}}}-$ KUBC & 0.51 \\
\hline$\overline{k^{\mathrm{app}}}-\mathrm{MBC}$ & 0.77 \\
\hline$\overline{\mu^{\mathrm{app}}}-$ KUBC & 0.64 \\
\hline$\overline{\mu^{\mathrm{app}}}-\mathrm{MBC}$ & 0.66 \\
\hline$\overline{V_{V}^{f}}$ & 0.67 \\
\hline
\end{tabular}

Table 4.8 RVE sizes estimated from computations with $\gamma=\frac{2}{3}, \epsilon_{\text {rel }}=5 \%$ and $n=1$

\begin{tabular}{l|l|l|l|l}
\hline$Z$ & BC & $K$ & $V_{\text {RVE }}$ & $\bar{N}$ \\
\hline$V_{V}$ & - & $4.4 \times 10^{-1}$ & $164^{3}$ & $2.1 \times 10^{3}$ \\
\hline$k^{\text {app }}$ & KUBC & $5.0 \times 10^{8}$ & $188^{3}$ & $2.8 \times 10^{3}$ \\
\hline$k^{\text {app }}$ & MBC & $3.5 \times 10^{8}$ & $296^{3}$ & $6.9 \times 10^{3}$ \\
\hline$\mu^{\text {app }}$ & KUBC & $4.0 \times 10^{8}$ & $245^{3}$ & $4.8 \times 10^{3}$ \\
\hline$\mu^{\text {app }}$ & MBC & $1.8 \times 10^{8}$ & $489^{3}$ & $1.9 \times 10^{4}$ \\
\hline
\end{tabular}

equal to 1 , similarly to the simulations in [105]. For the apparent shear modulus, $\gamma$ fluctuates between 0.64 and 0.66 . Hence, as a 1 st-order approximation, these physical properties may depend on the indicator function of the fibers. The same conclusion can be drawn for the apparent bulk modulus, but with further variation since $\gamma$ varies between 0.51 and 0.77 depending on the BC.

Values for RVE size and relative error in Table 4.8 can be compared to the volume considered and associated relative error in Table 4.6: for instance, if one considers the experimental error associated with the estimate of $\overline{k^{\text {app }}}$ using KUBC for $V=100^{3}$, with $n=13$ realizations, $\epsilon_{\text {rel }}=2.6 \%$. For the same relative error and number of realizations, the asymptotic model, on which values in Table 4.8 are based, yields an RVE size of $V=100.1^{3}$, which corresponds to an error of $0.1 \%$. The model is thus appropriate for every data point considered, except in the case of volumes for which boundary layer effects are not negligible.

\subsubsection{Conclusions}

This section questions the applicability of the concept of RVE for pathological cases such as Poisson fibers. The microstructural model was developed and implemented numerically. Hundreds of realizations were computed using FE, generating a large amount of data to be analyzed statistically. Overall, the method proposed in [114] and generalized in [57] for determining RVE sizes can be considered robust and effective, even for pathological microstructures. This is method is thus very useful for the homogenization of random architectured materials. 


\subsection{Conclusions}

The use of architectured materials in industrial applications is conditional upon the development of appropriate models for such materials. The present chapter covered the most critical aspects of computational homogenization for architectured materials. First, an introduction to classical computational homogenization was made and a description of a computational approach using the finite element method was given. This approach was applied for the specific case of periodic auxetic microstructures. Periodic homogenization elastoplastic constitutive materials was then explored. A systematic statistical approach for the computational homogenization of random architectured materials was presented. The problem of representativity is also critical when considering architectured materials in which the scale separation assumption becomes invalid, i.e. for finite structures made of architectured materials. Recent analytical results show that effective properties can indeed be determined independently of the number of unit cells $[30,160]$. The polynomial approach was developed further in the works of $[12,102,103]$. This approach was applied for modeling the homogenized behavior of auxetics [16] and 3D woven textiles based on experiments [170]. However the dependence on the choice of the representative unit cell remains without solution. Approaches like multifield methods [186] and computational continua $[73,74]$ may be appropriate alternatives to the polynomial approach. For more details about the mechanics of generalized continua, see $[5,75,180]$. The study of structures made of architectured materials has been the object of intensive research in the last few years [168], and has resulted in promising results concerning the development of architectured metamaterials for acoustic [175, 177] and elastodynamic applications [174, 176].

\section{References}

1. M. Abouaf, J.-L. Chenot, G. Raisson, P. Bauduin, Finite element simulation of hot isostatic pressing of metal powders. Int. J. Numer. Methods Eng. 25, 191-212 (1988)

2. A. Alderson, K.L. Alderson, D. Attard, K.E. Evans, R. Gatt, J.N. Grima, W. Miller, N. Ravirala, C.W. Smith, K. Zied, Elastic constants of 3-, 4- and 6-connected chiral and anti-chiral honeycombs subject to uniaxial in-plane loading. Compos. Sci. Technol. 70(7), 1042-1048 (2010)

3. G. Allaire, Shape Optimization by the Homogenization Method (Springer, Berlin, 2002)

4. R.F. Almgren, An isotropic three-dimensional structure with Poisson's ratio-1. J. Elast. 15, 427-430 (1985)

5. H. Altenbach, V. Eremeyev (eds.), Generalized Continua from the Theory to Engineering Applications (Springer, Berlin, 2013)

6. H. Altendorf, D. Jeulin, F. Willot, Influence of the fiber geometry on the macroscopic elastic and thermal properties. Int. J. Solids Struct. 51(23-24), 3807-3822 (2014)

7. J.C. Alvarez Elipe, A. Diaz Lantada, Comparative study of auxetic geometries by means of computer-aided design and engineering. Smart Mater. Struct. 21, 105004 (2012)

8. E. Andreassen, B. Lazarov, O. Sigmund, Design of manufacturable $3 \mathrm{~d}$ extremal elastic microstructure. Mech. Mater. 69(1), 1-10 (2014) 
9. U. Andreaus, F. dell'Isola, I. Giorgio, L. Placidi, T. Lekszycki, N.L. Rizzi, Numerical simulations of classical problems in two-dimensional (non) linear second gradient elasticity. Int. J. Eng. Sci. 108, 34-50 (2016)

10. A. Asadpoure, M. Tootkaboni, L. Valdevit, Topology optimization of multiphase architected materials for energy dissipation. Comput. Methods Appl. Mech. Eng. 325, 314-329 (2017)

11. M.F. Ashby, Y. Bréchet, Designing hybrid materials. Acta Mater. 51, 5801-5821 (2003)

12. N. Auffray, Analytical expressions for odd-order anisotropic tensor dimension. C. R. Mécaniques 342(5), 284-291 (2014)

13. N. Auffray, J. Dirrenberger, G. Rosi, A complete description of bi-dimensional anisotropic strain-gradient elasticity. Int. J. Solids Struct. 69-70, 195-210 (2015)

14. J.-L. Auriault, Heterogeneous medium is an equivalent macroscopic description possible? Int. J. Eng. Sci. 29(7), 785-795 (1991)

15. S. Babaee, J. Shim, J. Weaver, E. Chen, N. Patel, K. Bertoldi, 3d soft metamaterials with negative poisson's ratio. Adv. Mater. 25(36), 5044-5049 (2013)

16. A. Bacigalupo, L. Gambarotta, Homogenization of periodic hexa- and tetrachiral cellular solids. Compos. Struct. 116, 461-476 (2014)

17. X. Badiche, S. Forest, T. Guibert, Y. Bienvenu, J.-D. Bartout, P. Ienny, M. Croset, H. Bernet, Mechanical properties and non-homogeneous deformation of open-cell nickel foams: application of the mechanics of cellular solids and of porous materials. Mater. Sci. Eng. A 289, 276-288 (2000)

18. E. Bafekrpour, A. Dyskin, E. Pasternak, A. Molotnikov, Y. Estrin, Internally architectured materials with directionally asymmetric friction. Sci. Rep. 5, 10732 (2015)

19. E. Bafekrpour, A. Molotnikov, J.C. Weaver, Y. Brechet, Y. Estrin, Responsive materials: a novel design for enhanced machine-augmented composites. Sci. Rep. 4, 3783 (2014)

20. C. Barbier, R. Dendievel, D. Rodney, Numerical study of 3D-compressions of entangled materials. Comput. Mater. Sci. 45, 593-596 (2009a)

21. C. Barbier, R. Dendievel, D. Rodney, Role of friction in the mechanics of nonbounded fibrous materials. Phys. Rev. E 80(1), 016115 (2009b)

22. S. Bargmann, B. Klusemann, J. Markmann, J.E. Schnabel, K. Schneider, C. Soyarslan, J. Wilmers, Generation of $3 \mathrm{~d}$ representative volume elements for heterogeneous materials: a review. Prog. Mater. Sci. 96, 322-384 (2018)

23. R.J. Bathurst, L. Rothenburg, Note on a random isotropic granular material with negative Poisson's ratio. Int. J. Eng. Sci. 26(4), 373-383 (1988)

24. M. Bendsøe, O. Sigmund, Topology Optimization (Springer, Berlin, 2004)

25. I. Benedetti, F. Barbe, Modelling polycrystalline materials: an overview of three-dimensional grain-scale mechanical models. J. Multiscale Model. 5(1), 1350002 (2013)

26. M.J. Beran, Statistical Continuum Theories (Wiley, Hoboken, 1968)

27. C. Berdin, Z.Y. Yao, S. Pascal, Internal stresses in polycrystalline zirconia: microstructure effects. Comput. Mater. Sci. 70, 140-144 (2013)

28. K. Bertoldi, P. Reis, S. Willshaw, T. Mullin, Negative poisson's ratio behavior induced by an elastic instability. Adv. Mater. 22(3), 361-366 (2010)

29. J. Besson, G. Cailletaud, J.-L. Chaboche, S. Forest, M. Blétry, Non-Linear Mechanics of Materials, Volume 167 of Solid Mechanics and Its Applications (Springer, Berlin, 2010)

30. D. Bigoni, W. Drugan, Analytical derivation of cosserat moduli via homogenization of heterogeneous elastic materials. J. Appl. Mech. 74, 741-753 (2007)

31. A. Bironeau, J. Dirrenberger, C. Sollogoub, G. Miquelard-Garnier, S. Roland, Evaluation of morphological representative sample sizes for nanolayered polymer blends. J. Microsc. 264(1), 48-58 (2016)

32. M. Bornert, T. Bretheau, P. Gilormini, Homogénéisation en mécanique des matériaux, Tome 1: Matériaux aléatoires élastiques et milieux périodiques. (Hermès, 2001)

33. O. Bouaziz, Y. Bréchet, J.D. Embury, Heterogeneous and architectured materials: a possible strategy for design of structural materials. Adv. Eng. Mater. 10(1-2), 24-36 (2008)

34. O. Bouaziz, J.P. Masse, S. Allain, L. Orgéas, P. Latil, Compression of crumpled aluminum thin foils and comparison with other cellular materials. Mater. Sci. Eng. Struct. Mater. Prop. Microstruct. Process. 570, 1-7 (2013) 
35. Y. Bréchet, J.D. Embury, Architectured materials: expanding materials space. Scripta Mater. 68(1), 1-3 (2013)

36. H.J. Bunge, Texture Analysis in Materials Science (Butterworths, 1982)

37. B.D. Caddock, K.E. Evans, Microporous materials with negative Poisson's ratios: I. Microstructure and mechanical properties. J. Phys. D: Appl. Phys. 22, 1877-1882 (1989)

38. G. Cailletaud, S. Forest, D. Jeulin, F. Feyel, I. Galliet, V. Mounoury, S. Quilici, Some elements of microstructural mechanics. Comput. Mater. Sci. 27, 351-374 (2003)

39. G. Cailletaud, D. Jeulin, P. Rolland, Size effect on elastic properties of random composites. Eng. Comput. 11(2), 99-110 (1994)

40. O. Caty, E. Maire, R. Bouchet, Fatigue of metal hollow spheres structures. Adv. Eng. Mater. 10(3), 179-184 (2008)

41. V.J. Challis, A.P. Roberts, A.H. Wilkins, Design of three dimensional isotropic microstructures for maximized stiffness and conductivity. Int. J. Solids Struct. 45, 4130-4146 (2008)

42. C. Chateau, L. Gélébart, M. Bornert, J. Crépin, D. Caldemaison, Multiscale approach of mechanical behaviour of sic/sic composites: elastic behaviour at the scale of the tow. Tech. Mechanik 30(1-3), 45-55 (2010)

43. I. Chekkal, M. Bianchi, C. Remillat, F.-X. Becot, L. Jaouen, F. Scarpa, Vibro-acoustic properties of auxetic open cell foam: model and experimental results. Acta Acustica united Acustica 96(2), 266-274 (2010)

44. C.P. Chen, R.S. Lakes, Micromechanical analysis of dynamic behavior of conventional and negative Poisson's ratio foams. J. Eng. Mater. Technol. 118(3), 285-288 (1996)

45. Y. Chen, X.N. Liu, G.K. Hu, Q.P. Sun, Q.S. Zheng, Micropolar continuum modelling of bidimensional tetrachiral lattices. Proc. R. Soc. A Math. Phys. Eng. Sci. 470(2165), 20130734 (2014)

46. J.B. Choi, R.S. Lakes, Design of a fastener based on negative Poisson's ratio foam. Cell. Polym. 10(3), 205-212 (1991)

47. L. Courtois, E. Maire, M. Perez, D. Rodney, O. Bouaziz, Y. Bréchet, Mechanical properties of monofilament entangled materials. Adv. Eng. Mater. 14(12), 1128-1133 (2012)

48. A.S. Dalaq, D.W. Abueidda, R.K.A. Al-Rub, I.M. Jasiuk, Finite element prediction of effective elastic properties of interpenetrating phase composites with architectured $3 \mathrm{~d}$ sheet reinforcements. Int. J. Solids Struct. 83, 169-182 (2016)

49. L. Decker, D. Jeulin, I. Tovena, 3d morphological analysis of the connectivity of a porous medium. Acta Stereologica 17(1), 107-112 (1998)

50. C. Delisée, D. Jeulin, F. Michaud, Morphological characterization and porosity in 3D of cellulosic fibrous materials. C.-R. de l'Acad. des Sci. Serie IIb : Mécanique 329(3), 179-185 (2001)

51. F. dell'Isola, A. Della Corte, I. Giorgio, Higher-gradient continua: The legacy of piola, mindlin, sedov and toupin and some future research perspectives. Math. Mech. Solids 22(4), 852-872 (2017)

52. V.S. Deshpande, N.A. Fleck, Isotropic constitutive models for metallic foams. J. Mech. Phys. Solids 48, 1253-1283 (2000)

53. F. di Paola, Modélisation multi-échelles du comportement thermo-élastique de composites à particules sphériques. Ph.D. thesis, Ecole Centrale Paris, 2010

54. J. Dirrenberger, Effective properties of architectured materials. Ph.D. thesis, MINESParisTech, Paris, 2012

55. J. Dirrenberger, S. Forest, D. Jeulin, Elastoplasticity of auxetic materials. Comput. Mater. Sci. 64, 57-61 (2012)

56. J. Dirrenberger, S. Forest, D. Jeulin, Effective elastic properties of auxetic microstructures: anisotropy and structural applications. Int. J. Mech. Mater. Des. 9(1), 21-33 (2013)

57. J. Dirrenberger, S. Forest, D. Jeulin, Towards gigantic RVE sizes for stochastic fibrous networks. Int. J. Solids Struct. 51(2), 359-376 (2014)

58. J. Dirrenberger, S. Forest, D. Jeulin, C. Colin, Homogenization of periodic auxetic materials, in 11th International Conference on the Mechanical Behavior of Materials (ICM11) Procedia Engineering, vol. 10, pp. 1847-1852 (2011) 
59. L. Djumas, A. Molotnikov, G.P. Simon, Y. Estrin, Enhanced mechanical performance of bio-inspired hybrid structures utilising topological interlocking geometry. Sci. Rep. 6, 26706 (2016)

60. L. Djumas, G.P. Simon, Y. Estrin, A. Molotnikov, Deformation mechanics of non-planar topologically interlocked assemblies with structural hierarchy and varying geometry. Sci. Rep. 7(1), 11844 (2017)

61. W.J. Drugan, J.R. Willis, A micromechanics-based nonlocal constitutive equation and estimates of representative volume element size for elastic composites. J. Mech. Phys. Solids 44(4), 497-524 (1996)

62. A.V. Dyskin, Y. Estrin, A.J. Kanel-Belov, E. Pasternak, Toughening by fragmentation-how topology helps. Adv. Eng. Mater. 3, 885-888 (2001)

63. D. Embury, O. Bouaziz, Steel-based composites: driving forces and classifications. Ann. Rev. Mater. Res. 40, 213-241 (2010)

64. J. Escoda, D. Jeulin, F. Willot, C. Toulemonde, Three-dimensional morphological modelling of concrete using multiscale poisson polyhedra. J. Microsc. 258(1), 31-48 (2015)

65. Y. Estrin, A.V. Dyskin, E. Pasternak, Topological interlocking as a material design concept. Mater. Sci. Eng. C 31, 1189-1194 (2011)

66. Y. Estrin, A.V. Dyskin, E. Pasternak, H.C. Khor, A.J. Kanel-Belov, Topological interlocking of protective tiles for the space shuttle. Philos. Mag. Lett. 83, 351-355 (2003)

67. K.E. Evans, The design of doubly curved sandwich panels with honeycomb cores. Compos. Struct. 17(2), 95-111 (1991)

68. K.E. Evans, A. Alderson, Auxetic materials: Functional materials and structures from lateral thinking!. Adv. Mater. 12(9), 617-628 (2000)

69. K.E. Evans, M.A. Nkansah, I.J. Hutchinson, S.C. Rogers, Molecular network design. Nature 353, 124 (1991)

70. A. Fallet, P. Lhuissier, L. Salvo, Y. Bréchet, Mechanical behaviour of metallic hollow spheres foam. Adv. Eng. Mater. 10(9), 858-862 (2008)

71. A. Faure, G. Michailidis, G. Parry, N. Vermaak, R. Estevez, Design of thermoelastic multimaterial structures with graded interfaces using topology optimization. Struct. Multi. Optim. 56(4), 823-837 (2017)

72. Y. Feng, T. Siegmund, E. Habtour, J. Riddick, Impact mechanics of topologically interlocked material assemblies. Int. J. Impact Eng. 75, 140-149 (2015)

73. J. Fish, V. Filonova, D. Fafalis, Computational continua revisited. Int. J. Numer. Methods Eng. 102(3-4), 332-378 (2015)

74. J. Fish, S. Kuznetsov, Computational continua. Int. J. Numer. Methods Eng. 84, 774-802 (2010)

75. S. Forest, Mechanics of Generalized Continua and Heterogeneous Materials (Les Presses de 1Ecole des Mines de Paris, 2005)

76. S. Forest, J.-S. Blazy, Y. Chastel, F. Moussy, Continuum modeling of strain localization phenomena in metallic foams. J. Mater. Sci. 40, 5903-5910 (2005)

77. D. François, A. Pineau, A. Zaoui, Mechanical Behaviour of Materials, Volume 1: Micro- and Macroscopic Constitutive Behaviour, volume 180 of Solid Mechanics and Its Applications (Springer, Berlin, 2012)

78. A.J. Freeman, Materials by design and the exciting role of quantum computation/simulation. J. Comput. Appl. Math. 149(1), 27-56 (2002)

79. F. Fritzen, S. Forest, D. Kondo, T. Böhlke, Computational homogenization of porous materials of green type. Comput. Mech. 52(1), 121-134 (2013)

80. N. Gaspar, X.J. Ren, C.W. Smith, J.N. Grima, K.E. Evans, Novel honeycombs with auxetic behaviour. Acta Mater. 53, 2439-2445 (2005)

81. M.G.D. Geers, J. Yvonnet, Multiscale modeling of microstructure property relations. MRS Bull. 41(8), 610-616 (2016)

82. L. Gélébart, C. Chateau, M. Bornert, Conditions aux limites mixtes normales. In 19ème Congrès Français de Mécanique, 24-28 August 2009, Marseille (2009) 
83. A. Ghaedizadeh, J. Shen, X. Ren, Y.M. Xie, Tuning the performance of metallic auxetic metamaterials by using buckling and plasticity. Materials 9(54), 1-17 (2016)

84. I.M. Gitman, H. Askes, L.J. Sluys, Representative volume: Existence and size determination. Eng. Fract. Mech. 74, 2518-2534 (2007)

85. R.J. Green, A plasticity theory for porous solids. International J. Mech. Sci. 14, 215-224 (1972)

86. J.K. Guest, J.H. Prévost, Optimizing multifunctional materials: design of microstructures for maximized stiffness and fluid permeability. Int. J. Solids Struct. 43(22-23), 7028-7047 (2006)

87. L. Guiducci, P. Fratzl, Y. Bréchet, J. Dunlop, Pressurized honeycombs as soft-actuators: a theoretical study. J. R. Soc. Interface 11, 20140458 (2014)

88. Z. Hashin, Analysis of composite materials-a survey. J. Appl. Mech. 50, 481-505 (1983)

89. H. Hatami-Marbini, R.C. Picu, Heterogeneous long-range correlated deformation of semiflexible random fiber networks. Phys. Rev. E 80(4) (2009)

90. S. Hazanov, Hill condition and overall properties of composites. Arch. Appl. Mech. 68, 385394 (1998)

91. S. Hazanov, C. Huet, Order relationships for boundary conditions effect in heterogeneous bodies smaller than the representative volume. J. Mech. Phys. Solids 42(12), 1995-2011 (1994)

92. J. Henry, S. Pimenta, Increasing damage tolerance in composites using hierarchical brickand-mortar microstructures. J. Mech. Phys. Solids 118, 322-340 (2018)

93. C.T. Herakovich, Composite laminates with negative through-the-thickness poisson's ratios. J. Compos. Mater. 18(5), 447-455 (1984)

94. R. Hill, Elastic properties of reinforced solids: Some theoretical principles. J. Mech. Phys. Solids 11, 357-372 (1963)

95. R. Hill, The essential structure of constitutive laws for metal composites and polycrystals. J. Mech. Phys. Solids 15, 79-95 (1967)

96. J.B. Hopkins, L.A. Shaw, T.H. Weisgraber, G.R. Farquar, C.D. Harvey, C.M. Spadaccini, Design of nonperiodic microarchitectured materials that achieve graded thermal expansions. J. Mech. Rob. 8(5), 051010 (2016)

97. A. Hor, N. Saintier, C. Robert, T. Palin-Luc, F. Morel, Statistical assessment of multiaxial hcf criteria at the grain scale. Int. J. Fatigue 67, 151-158 (2014)

98. C. Huet, Application of variational concepts to size effects in elastic heterogeneous bodies. J. Mech. Phys. Solids 38(6), 813-841 (1990)

99. C. Huet, An integrated micromechanics and statistical continuum thermodynamics approach for studying the fracture behaviour of microcracked heterogeneous materials with delayed response. Eng. Fracture Mech. 58(5-6), 459-556 (1997)

100. T.P. Hughes, A. Marmier, K.E. Evans, Auxetic frameworks inspired by cubic crystals. Int. J. Solids Struct. 47, 1469-1476 (2010)

101. A. Iltchev, V. Marcadon, S. Kruch, S. Forest, Computational homogenisation of periodic cellular materials: application to structural modelling. Int. J. Mech. Sci. 93, 240-255 (2015)

102. R. Jänicke, H. Sehlhorst, A. Dster, S. Diebels, Micromorphic two-scale modelling of periodic grid structures. Int. J. Multiscale Comput. Eng. 11, 161-176 (2013)

103. R. Jänicke, H. Steeb, Minimal loading conditions for higher-order numerical homogenisation schemes. Arch. Appl. Mech. 82(8), 1075-1088 (2012)

104. A. Jean, G.C. Engelmayr, Finite element analysis of an accordion-like honeycomb scaffold for cardiac tissue engineering. J. Biomech. 43, 3035-3043 (2010)

105. A. Jean, D. Jeulin, S. Forest, S. Cantournet, F. N'Guyen, A multiscale microstructure model of carbon black distribution in rubber. J. Microsc. 241(3), 243-260 (2011a)

106. A. Jean, F. Willot, S. Cantournet, S. Forest, D. Jeulin, Large-scale computations of effective elastic properties of rubber with carbon black fillers. Int. J. Multiscale Comput. Eng. 9(3), 271-303 (2011b)

107. D. Jeulin, Modèles de fonctions aléatoires multivariables. Sci. de la Terre 30, 225-256 (1991)

108. D. Jeulin, Random texture models for material structures. Stat. Comput. 10(2), 121-132 (2000) 
109. D. Jeulin, Caractérisation Morphologique et Modèles de Structures Aléatoires, volume 1 of Homogénéisation en Mécanique des Matériaux, chapter 4, pp. 95-132. Hermès (2001)

110. D. Jeulin, Variance scaling of Boolean random varieties. Technical report, Centre de Morphologie Mathématique. N/10/11/MM (2011), hal-00618967, version 1 (2011)

111. D. Jeulin, Power laws variance scaling of Boolean random varieties. Methodol. Comput. Appl. Probab., pp. 1-15 (2015)

112. D. Jeulin, M. Ostoja-Starzewski, Mechanics of Random and Multiscale Microstructures (Springer, CISM Courses, 2001)

113. N. Kaminakis, G. Drosopoulos, G. Stavroulakis, Design and verification of auxetic microstructures using topology optimization and homogenization. Arch. App. Mech. 85(9), 1289-1306 (2015)

114. T. Kanit, S. Forest, I. Galliet, V. Mounoury, D. Jeulin, Determination of the size of the representative volume element for random composites: Statistical and numerical approach. Int. J. Solids Struct. 40, 3647-3679 (2003)

115. T. Kanit, F. N'Guyen, S. Forest, D. Jeulin, M. Reed, S. Singleton, Apparent and effective physical properties of heterogeneous materials: representativity of samples of two materials from food industry. Comput. Methods Appl. Mech. Eng. 195, 3960-3982 (2006)

116. S. Khakalo, J. Niiranen, Isogeometric analysis of higher-order gradient elasticity by user elements of a commercial finite element software. Comput.-Aided Des. 82, 154-169 (2017)

117. S. Khandelwal, T. Siegmund, R.J. Cipra, J.S. Bolton, Adaptive mechanical properties of topologically interlocking material systems. Smart Mater. Struct. 24(4), 045037 (2015)

118. A. Kolopp, S. Rivallant, C. Bouvet, Experimental study of sandwich structures as armour against medium-velocity impacts. Int. J. Impact Eng. 61, 24-35 (2013)

119. C. Körner, Y. Liebold-Ribeiro, A systematic approach to identify cellular auxetic materials. Smart Mater. Struct. 24(2), 025013 (2015)

120. M. Kotani, S. Ikeda, Materials inspired by mathematics. Sci. Technol. Adv. Mater. 17(1), 253-259 (2016)

121. N. Kowalski, L. Delannay, P. Yan, J.F. Remacle, Finite element modeling of periodic polycrystalline aggregates with intergranular cracks. Int. J. Solids Struct. 90, 60-68 (2016)

122. V. Krasavin, A. Krasavin, Auxetic properties of cubic metal single crystals. Phys. Status Solidi b 251(11), 2314-2320 (2014)

123. T. Krause, A. Molotnikov, M. Carlesso, J. Rente, K. Rezwan, Y. Estrin, D. Koch, Mechanical properties of topologically interlocked structures with elements produced by freeze gelation of ceramic slurries. Adv. Eng. Mater. 14(5), 335-341 (2012)

124. R.S. Lakes, Foam structures with a negative Poisson's Ratio. Science 235, 1038-1040 (1987)

125. R.S. Lakes, Deformation mechanisms in negative Poisson's ratio materials: structural aspects. J. Mater. Sci. 26, 2287-2292 (1991)

126. C. Lantuéjoul, Ergodicity and integral range. J. Microsc. 161, 387-403 (1991)

127. C. Lantuéjoul, Geostatistical Simulation: Models and Algorithms (Springer, Berlin, 2002)

128. L. Laszczyk, R. Dendievel, O. Bouaziz, Y. Br'echet, G. Parry, Design of architectured sandwich core materials using topological optimization methods. In Symposium LL Architectured Multifunctional Materials, volume 1188 of MRS Proceedings (2009)

129. A. Lebée, K. Sab, Homogenization of thick periodic plates: application of the bending-gradient plate theory to a folded core sandwich panel. Int. J. Solids Struct. 49(19-20), 2778-2792 (2012)

130. M. Lewandowski, M. Amiot, A. Perwuelz, Development and characterization of 3D nonwoven composites. Mater. Sci. Forum 714, 131-137 (2012)

131. A.W. Lipsett, A.I. Beltzer, Reexamination of dynamic problems of elasticity for negative poisson's ratio. J. Acoust. Soc. Am. 84(6), 2179-2186 (1988)

132. J. Liu, T. Gu, S. Shan, S.H. Kang, J.C. Weaver, K. Bertoldi, Harnessing buckling to design architected materials that exhibit effective negative swelling. Adv. Mater. 28(31), 6619-6624 (2016)

133. K. Madi, S. Forest, M. Boussuge, S. Gailliègue, E. Lataste, J.-Y. Buffière, D. Bernard, D. Jeulin, Finite element simulations of the deformation of fused-cast refractories based on $\mathrm{X}$ ray computed tomography. Comput. Mater. Sci. 39, 224-229 (2007) 
134. K. Madi, S. Forest, P. Cordier, M. Boussuge, Numerical study of creep in two-phase aggregates with a large rheology contrast: implications for the lower mantle. Earth Planet. Sci. Lett. 237(1-2), 223-238 (2005)

135. J. Martin, J.-J. Heyder-Bruckner, C. Remillat, F. Scarpa, K. Potter, M. Ruzzene, The hexachiral prismatic wingbox concept. Phys. Status Solidi (b) 245(3), 570-577 (2008)

136. A. Mather, R. Cipra, T. Siegmund, Structural integrity during remanufacture of a topologically interlocked material. Int. J. Struct. Integrity 3(1), 61-78 (2012)

137. G. Matheron, The Theory of Regionalized Variables and its Applications Les Cahiers du Centre de Morphologie Mathématique de Fontainebleau. (Ecole des Mines de Paris, 1971)

138. G. Matheron, Random Sets and Integral Geometry (Wiley, 1975)

139. G. Matheron, Estimating and Choosing (Springer, Berlin, 1989)

140. K. Matouš, M.G.D. Geers, V.G. Kouznetsova, A. Gillman, A review of predictive nonlinear theories for multiscale modeling of heterogeneous materials. J. Comput. Phys. 330, 192-220 (2017)

141. L. Mezeix, C. Bouvet, J. Huez, D. Poquillon, Mechanical behavior of entangled fibers and entangled cross-linked fibers during compression. J. Mater. Sci. 44(14), 3652-3661 (2009)

142. J.-C. Michel, H. Moulinec, P. Suquet, Effective properties of composite materials with periodic microstructure: a computational approach. Comput. Methods Appl. Mech. Eng. 172, 109-143 (1999)

143. R.E. Miller, A continuum plasticity model for the constitutive and indentation behaviour of foamed metals. Int. J. Mech. Sci. 42, 729-754 (2000)

144. G.W. Milton, Composite materials with Poisson's Ratios close to -1. J. Mech. Phys. Solids 40(5), 1105-1137 (1992)

145. A. Molotnikov, Y. Estrin, A.V. Dyskin, E. Pasternak, A.J. Kanel-Belov, Percolation mechanism of failure of a planar assembly of interlocked osteomorphic elements. Eng. Fracture Mech. 74, 1222-1232 (2007)

146. A. Molotnikov, R. Gerbrand, O. Bouaziz, Y. Estrin, Sandwich panels with a core segmented into topologically interlocked elements. Adv. Eng. Mater. 15(8), 728-731 (2013)

147. A. Molotnikov, R. Gerbrand, Y. Qi, G.P. Simon, Y. Estrin, Design of responsive materials using topologically interlocked elements. Smart Mater. Struct. 24(2), 025034 (2015)

148. G.B. Olson, Beyond discovery: design for a new material world. Calphad 25(2), 175-190 (2001)

149. M. Osanov, J.K. Guest, Topology optimization for architected materials design. Ann. Rev. Mater. Res. 46, 211-233 (2016)

150. M. Ostoja-Starzewski, Microstructural randomness versus representative volume element in thermomechanics. J. Appl. Mech. 69(1), 25-35 (2002)

151. M. Ostoja-Starzewski, Microstructural Randomness and Scaling in Mechanics of Materials (Chapman \& Hall/CRC, Mordern Mechanics and Mathematics, 2008)

152. M. Oumarou, D. Jeulin, J. Renard, Etude statistique multi-échelle du comportement élastique et thermique d'un composite thermoplastique. Rev. des Compos. et des matériaux avancés 21, 221-254 (2011)

153. M. Oumarou, D. Jeulin, J. Renard, P. Castaing, Multi-scale statistical approach of the elastic and thermal behavior of a thermoplastic polyamid-glass fiber composite. Tech. Mechanik 32(2-5), 484-506 (2012)

154. D.H. Pahr, P.K. Zysset, Influence of boundary conditions on computed apparent elastic properties of cancellous bone. Biomech. Model. Mechanobiol. 7, 463-476 (2008)

155. E. Pasternak, A. Dyskin, Materials and structures with macroscopic negative poissons ratio. Int. J. Eng. Sci. 52, 103-114 (2012)

156. C. Pelissou, J. Baccou, Y. Monerie, F. Perales, Determination of the size of the representative volume element for random quasi-brittle composites. Int. J. Solids Struct. 46, 2842-2855 (2009)

157. X.L. Peng, E. Husser, G.Y. Huang, S. Bargmann, Modeling of surface effects in crystalline materials within the framework of gradient crystal plasticity. J. Mech. Phys. Solids 112, 508$522(2018)$ 
158. C. Peyrega, D. Jeulin, C. Delisée, J. Malvestio, 3D morphological modelling of a random fibrous network. Image Anal. Stereol. 28, 129-141 (2009)

159. C. Peyrega, D. Jeulin, C. Delisée, J. Malvestio, 3D morphological characterization of phonic insulation fibrous media. Adv. Eng. Mater. 13(3), 156-164 (2011)

160. Pham, T. T.T, Un modèle d'endommagement à gradient de déformation à partir de la méthode d'homogénéisation pour les matériaux fragiles. Ph.D. thesis, Université Paris XIII, 2010

161. R.C. Picu, Mechanics of random fiber networks-a review. Soft Matter 7, 6768-6785 (2011)

162. R.C. Picu, H. Hatami-Marbini, Long-range correlations of elastic fields in semi-flexible fiber networks. Comput. Mech. 46, 635-640 (2010)

163. E. Piollet, Amortissement non-linéaire des structures sandwichs à matériau d'âme en fibres enchevêtrées. Ph.D. thesis, ISAE, Toulouse, 2014

164. E. Piollet, G. Michon, D. Poquillon, Nonlinear vibration behavior of sandwich beams with entangled fiber core material. In ASME 2013 International Design Engineering Technical Conferences and Computers and Information in Engineering Conference (American Society of Mechanical Engineers, 2013), pp. V008T13A013-V008T13A013

165. E. Piollet, D. Poquillon, G. Michon, Dynamic hysteresis modelling of entangled cross-linked fibres in shear. J. Sound Vibr. 383, 248-264 (2016)

166. L. Placidi, E. Barchiesi, A. Della Corte, Mathematical Modelling in Solid Mechanics, volume 69 of Advanced Structured Materials, chapter Identification of Two-Dimensional Pantographic Structures with a Linear D4 Orthotropic Second Gradient Elastic Model Accounting for External Bulk Double Forces (Springer, Singapore, 2017), pp. 211-232

167. L. Placidi, A.R. El Dhaba, Semi-inverse method à la saint-venant for two-dimensional linear isotropic homogeneous second-gradient elasticity. Math. Mech. Solids 22(5), 919-937 (2015)

168. M. Poncelet, A. Somera, C. Morel, C. Jailin, N. Auffray, An experimental evidence of the failure of Cauchy elasticity for the overall modeling of a non-centro-symmetric lattice under static loading. Int. J. Solids Struct. 147, 223-237 (2018)

169. D. Prall, R.S. Lakes, Properties of a Chiral honeycomb with a Poisson's ratio of -1. Int. J. Mech. Sci. 39(3), 305-314 (1997)

170. Y. Rahali, I. Goda, J.-F. Ganghoffer, Numerical identification of classical and nonclassical moduli of $3 \mathrm{~d}$ woven textiles and analysis of scale effects. Compos. Struct. 135, 122-139 (2016)

171. X. Ren, J. Shen, A. Ghaedizadeh, H. Tian, Y.M. Xie, A simple auxetic tubular structure with tuneable mechanical properties. Smart Mater. Struct. 5(6), 065012 (2016)

172. A. Reuss, Berechnung der fließgrenze von mischkristallen auf grund der plastizitätsbedingung für einkristalle. Zeitschrift für angewandte Mathematik und Mechanik 9(1), 49-58 (1929)

173. D. Rodney, B. Gadot, O.R. Martinez, S.R. Du Roscoat, L. Orgéas, Reversible dilatancy in entangled single-wire materials. Nat. Mater. 15(1), 72 (2016)

174. G. Rosi, N. Auffray, Anisotropic and dispersive wave propagation within strain-gradient framework. Wave Motion 63, 120-134 (2016)

175. G. Rosi, V.H. Nguyen, A. Loseille, S. Naili, Ultrasonic characterization of porous gyroid scaffolds for bone tissue engineering: mechanical modelling and numerical validation. J. Acoust. Soc. Am. 144(3), 1854 (2018a)

176. G. Rosi, L. Placidi, N. Auffray, On the validity range of strain-gradient elasticity: a mixed static-dynamic identification procedure. Eur. J. Mech. A/Solids 69, 179-191 (2018b)

177. G. Rosi, I. Scala, V. Nguyen, S. Naili, Wave propagation in strain gradient poroelastic medium with microinertia: closed-form and finite element solutions. Zeitschrift für angewandte Mathematik und Physik 63(3), 58 (2017)

178. L. Rothenburg, A.A. Berlin, R.J. Bathurst, Microstructure of isotropic materials with negative Poisson's ratio. Nature 354, 470-472 (1991)

179. K. Sab, On the homogenization and the simulation of random materials. Eur. J. Mech. A/Solids 11(5), 585-607 (1992)

180. K. Sab, A. Lebée, Homogenization of Heterogeneous Thin and Thick Plates (Wiley, Hoboken, 2015) 
181. K. Sab, B. Nedjar, Periodization of random media and representative volume element size for linear composites. C.-R. de l'Acad. des Sci. Serie IIb : Mécanique 333, 187-195 (2005)

182. M. Salmi, F. Auslender, M. Bornert, M. Fogli, Apparent and effective mechanical properties of linear matrix-inclusion random composites: Improved bounds for the effective behavior. Int. J. Solids Struct. 49, 1195-1211 (2012a)

183. M. Salmi, F. Auslender, M. Bornert, M. Fogli, Various estimates of representative volume element sizes based on a statistical analysis of the apparent behavior of random linear composites.

C.-R. de l'Acad. des Sci. Serie IIb : Mécanique 340, 230-246 (2012b)

184. K. Salonitis, D. Chantzis, V. Kappatos, A hybrid finite element analysis and evolutionary computation method for the design of lightweight lattice components with optimized strut diameter. Int. J. Adv. Manufact. Technol. 90(9-12), 2689-2701 (2017)

185. E. Sanchez-Palencia, A. Zaoui, Homogenization Techniques for Composite Media, vol. 272. Lecture Notes in Physics (Springer, Berlin, 1987)

186. V. Sansalone, P. Trovalusci, F. Cleri, Multiscale modeling of composite materials by a multifield finite element approach. Int. J. Multiscale Comput. Eng. 3, 463-480 (2005)

187. B. Sarac, J. Wilmers, S. Bargmann, Property optimization of porous metallic glasses via structural design. Mater. Lett. 134, 306-310 (2014)

188. F. Scarpa, J.R. Yates, L.G. Ciffo, S. Patsias, Dynamic crushing of auxetic open-cell polyurethane foam. Proc. Inst. Mech. Eng. Part C-J. Mech. Eng. Sci. 216(12), 1153-1156 (2002)

189. S. Schaare, W. Riehemann, Y. Estrin, Damping properties of an assembly of topologically interlocked cubes. Mater. Sci. Eng. A Struct. Mater. Prop. Microstruct. Process. 521-522, 380-383 (2009)

190. T.A. Schaedler, W.B. Carter, Architected cellular materials. Ann. Rev. Mater. Res. 46, 187210 (2016)

191. T.A. Schaedler, A.J. Jacobsen, A. Torrents, A.E. Sorensen, J. Lian, J.R. Greer, L. Valdevit, W.B. Carter, Ultralight metallic microlattices. Science 334(6058), 962-965 (2011)

192. K. Schladitz, S. Peters, D. Reinel-Bitzer, A. Wiegmann, J. Ohser, Design of acoustic trim based on geometric modeling and flow simulation for non-woven. Comput. Mater. Sci. 38, 56-66 (2006)

193. I. Shufrin, E. Pasternak, A. Dyskin, Planar isotropic structures with negative poissons ratio. Int. J. Solids Struct. 49(17), 2239-2253 (2012)

194. I. Shufrin, E. Pasternak, A. Dyskin, Negative poissons ratio in hollow sphere materials. Int. J. Solids Struct. 54, 192-214 (2015)

195. P. Smith, S. Torquato, Computer simulation results for the two-point probability function of composite media. J. Comput. Phys. 76(1), 176-191 (1988)

196. M.A. Soare, R.C. Picu, An approach to solving mechanics problems for materials with multiscale self-similar microstructure. Int. J. Solids Struct. 44, 7877-7890 (2007)

197. C. Soyarslan, S. Bargmann, M. Pradas, J. Weissmuller, 3D stochastic bicontinuous microstructures: generation, topology and elasticity. Acta Mater. 149, 326-340 (2018)

198. A. Spadoni, M. Ruzzene, S. Gonella, F. Scarpa, Phononic properties of hexagonal chiral lattices. Wave Motion 46(7), 435-450 (2009)

199. J. Teixeira-Pinto, C. Nadot-Martin, F. Touchard, M. Gueguen, S. Castagnet, Towards the size estimation of a representative elementary domain in semi-crystalline polymers. Mechanics of Materials in press (2016)

200. K. Terada, M. Hori, T. Kyoya, N. Kikuchi, Simulation of the multi-scale convergence in computational homogenization approaches. Int. J. Solids Struct. 37, 2285-2311 (2000)

201. S. Torquato, Morphology and effective properties of disordered heterogeneous media. Int. J. Solids Struct. 35(19), 2385-2406 (1998)

202. S. Torquato, Random Heterogeneous Materials (Springer, Berlin, 2001)

203. D.K. Trinh, R. Jänicke, N. Auffray, S. Diebels, S. Forest, Evaluation of generalized continuum substitution models for heterogeneous materials. Int. J. Multiscale Comput. Eng. 10(6), 527$549(2012)$ 
204. N. Vermaak, G. Michailidis, G. Parry, R. Estevez, G. Allaire, Y. Brechet, Material interface effects on the topology optimizationof multi-phase structures using a level set method. Struct. Mult. Optim. 50(4), 623-644 (2014)

205. W.M. Vicente, Z.H. Zuo, R. Pavanello, T.K.L. Calixto, R. Picelli, Y.M. Xie, Concurrent topology optimization for minimizing frequency responses of two-level hierarchical structures. Comput. Methods Appl. Mech. Eng. 301, 116-136 (2016)

206. W. Voigt, Ueber die beziehung zwischen den beiden elasticitätsconstanten isotroper körper. Ann. der Phys. und Chem. 38, 573-587 (1889)

207. Y. Wang, H. Xu, D. Pasini, Multiscale isogeometric topology optimization for lattice materials. Comput. Methods Appl. Mech. Eng. 316, 568-585 (2017a)

208. Z.P. Wang, L.H. Poh, J. Dirrenberger, Y. Zhu, S. Forest, Isogeometric shape optimization of smoothed petal auxetic structures via computational periodic homogenization. Comput. Methods Appl. Mech. Eng. 323, 250-271 (2017b)

209. P.M. Weaver, M.F. Ashby, The optimal selection of material and section-shape. J. Eng. Des. 7(2), 129-150 (1996)

210. B. Xu, X. Huang, S.W. Zhou, Y.M. Xie, Concurrent topological design of composite thermoelastic macrostructure and microstructure with multi-phase material for maximum stiffness. Compos. Struct. 150, 84-102 (2016a)

211. S. Xu, J. Shen, S. Zhou, X. Huang, Y.M. Xie, Design of lattice structures with controlled anisotropy. Mater. Des. 93, 443-447 (2016b)

212. W. Yang et al., Review on auxetic materials. J. Mater. Sci. 39, 3269-3279 (2004)

213. C.L.Y. Yeong, S. Torquato, Reconstructing random media. Phys. Rev. E 57(1), 495 (1998) 